\title{
ARTICLE \\ Hierarchical activation of compartmentalized pools of AMPK depends on severity of nutrient or energy stress
}

Yue Zong ${ }^{1}$, Chen-Song Zhang ${ }^{1}$, Mengqi $\mathrm{Li}^{1}$, Wen Wang ${ }^{2,3}$, Zhichao Wang ${ }^{2,3}$, Simon A. Hawley ${ }^{4}$, Teng Ma ${ }^{1}$, Jin-Wei Feng ${ }^{1}$, Xiao Tian $^{1}$, Qu Qi ${ }^{1}$, Yu-Qing Wu ${ }^{1}$, Cixiong Zhang ${ }^{1}$, Zhiyun Ye ${ }^{1}$, Shu-Yong Lin $\mathbb{D}^{1}$, Hai-Long Piao ${ }^{2,3}$, D. Grahame Hardie ${ }^{4}$ and Sheng-Cai Lin ${ }^{1}$

AMPK, a master regulator of metabolic homeostasis, is activated by both AMP-dependent and AMP-independent mechanisms. The conditions under which these different mechanisms operate, and their biological implications are unclear. Here, we show that, depending on the degree of elevation of cellular AMP, distinct compartmentalized pools of AMPK are activated, phosphorylating different sets of targets. Low glucose activates AMPK exclusively through the AMP-independent, AXIN-based pathway in lysosomes to phosphorylate targets such as ACC1 and SREBP1C, exerting early anti-anabolic and pro-catabolic roles. Moderate increases in AMP expand this to activate cytosolic AMPK also in an AXIN-dependent manner. In contrast, high concentrations of AMP, arising from severe nutrient stress, activate all pools of AMPK independently of AXIN. Surprisingly, mitochondrion-localized AMPK is activated to phosphorylate ACC2 and mitochondrial fission factor (MFF) only during severe nutrient stress. Our findings reveal a spatiotemporal basis for hierarchical activation of different pools of AMPK during differing degrees of stress severity.

Cell Research (2019) 29:460-473; https://doi.org/10.1038/s41422-019-0163-6

\section{INTRODUCTION}

The AMP-activated protein kinase (AMPK) is a pivotal sensor for monitoring cellular nutrient supply and energy status, and plays crucial roles in adaptive responses to nutrient availability and falling energy levels. ${ }^{1-5}$ AMPK comprises a heterotrimeric complex of a catalytic a subunit and regulatory $\beta$ and $\gamma$ subunits. The $\gamma$ subunit provides binding sites for the regulatory nucleotides AMP, ADP and ATP, whose occupancy depends upon the cellular AMP: ATP and ADP:ATP ratios. ${ }^{6-8}$ Binding of AMP results in allosteric activation of AMPK whereas binding of AMP or ADP enhances the phosphorylation of Thr172 on the a subunit by the upstream kinase liver kinase B1 (LKB1) and inhibits Thr172 dephosphorylation by protein phosphatases. All of these three effects are opposed by the binding of ATP. ${ }^{9}$ As an alternative to this classical or canonical mechanism for activation, Thr172 can also be phosphorylated by a non-canonical, AMP/ADP-independent process by $\mathrm{Ca}^{2+} /$ calmodulin-dependent protein kinase kinase-2 (CaMKK2/CaMKK $\beta$ ) in response to increases in cellular $\mathrm{Ca}^{2+}$ concentration. ${ }^{10-12}$ Glucose starvation of cells has been known for many years to activate $\mathrm{AMPK}^{13}$ and was believed to occur exclusively via the canonical mechanism involving increases in cellular AMP or ADP. However, this view has been challenged by recent findings that AMPK located at the lysosome can be activated by falling levels of glucose, both in vivo and in vitro, via an additional AMP/ADP-independent mechanism. ${ }^{14,15}$ This mechanism requires the glycolytic enzyme fructose-1,6-bisphosphate (FBP) aldolase, the vacuolar $\mathrm{H}^{+}$-ATPase ( $v$-ATPase), the pentameric Ragulator complex (LAMTOR1-LAMTOR5), ${ }^{16,17}$ and the scaffold protein AXIN1 (often referred to simply as AXIN), which binds the upstream kinase LKB1. As glucose in the medium is reduced, aldolase associated with the v-ATPase becomes progressively unoccupied by $\mathrm{FBP}$, thus transmiting a glucose shortage signal to the v-ATPase:Ragulator complex. The latter then undergoes conformational changes that allow binding of the AXIN1LKB1 complex, which in turn forms a complex with AMPK at the lysosome. This "super-complex", referred to below as the lysosomal AMPK activation complex, brings LKB1 and AMPK together, leading to phosphorylation and activation of the latter. $^{4,14,18,19}$ In addition to the lysosomal AMPK, subcellular fractionation and fluorescence microscopy have revealed the localization and activation of AMPK in other locations such as the cytosol, nucleus and mitochondria, both in mammalian cells and in yeast. ${ }^{20-26}$ Importantly, N-myristoylation of AMPK- $\beta$ subunits ${ }^{27,28}$ has been shown to be necessary for lysosomal localization and activation of AMPK in an AMP-independent manner in response to glucose starvation, ${ }^{14}$ as well as for mitochondrial localization and induction of mitophagy in various mammalian cell lines. $^{24}$

Once activated, AMPK directly phosphorylates multiple targets involved in the regulation of metabolic processes to maintain energy homeostasis. These effects can be categorized by the inhibition of anabolism and stimulation of catabolism, thus minimizing ATP consumption and stimulating ATP production respectively. ${ }^{3}$ For example, AMPK phosphorylates and inactivates both isoforms of acetyl-CoA carboxylase, i.e. ACC1 (on Ser79) ${ }^{29}$ and ACC2 (on Ser221). ${ }^{30}$ These isoforms both catalyze the conversion of acetyl-CoA to malonyl-CoA, but ACC1 is located in the cytosol, and is proposed to provide the pool of malonyl-CoA

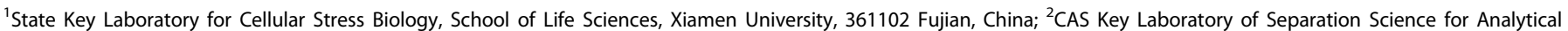

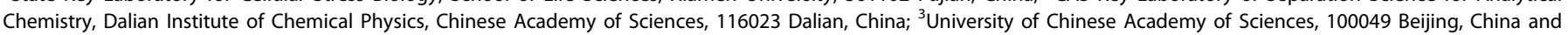
${ }^{4}$ Division of Cell Signalling and Immunology, College of Life Sciences, University of Dundee, DD1 5EH Dundee, Scotland, UK

Correspondence: Sheng-Cai Lin (linsc@xmu.edu.cn)

These authors contributed equally: Yue Zong, Chen-Song Zhang, Mengqi Li, Wen Wang
}

Received: 19 February 2019 Accepted: 15 March 2019

Published online: 4 April 2019 
for fatty acid synthesis, whereas ACC2 is located at the mitochondrion and is proposed to provide the pool of malonylCoA that inhibits carnitine:palmitoyl-CoA acyl transferase-1 (CPT1) and hence the uptake and subsequent oxidation of fatty acids in mitochondria. ${ }^{31-33}$ Therefore, AMPK can both inhibit fatty acid synthesis by phosphorylating ACC1 and promote fatty acid oxidation by inhibiting ACC2. ${ }^{34}$ AMPK also phosphorylates the endoplasmic reticulum-localized form of the transcription factor sterol regulatory element-binding protein-1c (SREBP-1c) at Ser372 to inhibit its proteolytic cleavage and thereby suppress fatty acid synthesis at the transcriptional level. ${ }^{35}$ It also inhibits other anabolic pathways such as glycogen, rRNA and nucleotide synthesis $^{34,36}$ and down-regulates mTORC 1 by phosphorylating its upstream negative regulator, tuberous sclerosis complex-2 (TSC2), ${ }^{37}$ and/or the mTORC1 subunit RAPTOR. ${ }^{38}$ Interestingly, when cells are facing a shortage of glucose, the lysosomal vATPase and Ragulator complex becomes involved in AMPK activation and dissociates mTORC1 from the lysosomal surface, providing another level of inhibitory regulation of mTORC1.39,40 Apart from blocking anabolic processes, AMPK stimulates various catabolic processes including glucose uptake and autophagy. For example, it phosphorylates TBC1D1 to promote glucose uptake in skeletal muscle. ${ }^{41,42}$ AMPK also directly phosphorylates ULK1 and Beclin-1 at multiple sites to initiate autophagy under energy stress. ${ }^{43-45}$ The mitochondrial outer membrane-localized mitochondrial fission factor (MFF), which promotes mitochondrial fragmentation prior to mitophagy, is also a direct substrate of AMPK, thus leading to induction of mitophagy. ${ }^{46,47}$ Moreover, recent evidence has indicated that AMPK is also localized in the nucleus, where it phosphorylates the ten-eleven translocation protein 2 (TET2). ${ }^{48,49}$

In this study, we have systemically evaluated how compartmentalized pools of AMPK are regulated, and how different subcellular targets of AMPK are phosphorylated in response to different severities of nutrient or energy stress. Emphasis was placed on the dependency of AMPK activation on increases in cellular AMP, and AXIN1 as the scaffold for the upstream kinase LKB1. We demonstrate that in MEFs starved for glucose or the livers of mice starved for $16 \mathrm{~h}$, when AMP:ATP or ADP:ATP ratios remained unchanged, AMPK activation occurs exclusively in the lysosomal pool. At medium-to-high AMP levels, as seen in HEK293 cells starved for glucose, or in MEFs undergoing early phases of severe nutrient starvation (removal of both glucose and glutamine from the medium), both cytosolic and lysosomal AMPK is activated, but AXIN1 is still needed to promote the association of LKB1-AMPK. By contrast, when cellular AMP levels are elevated to high levels due to severe nutrient starvation or ischemia, mitochondrial AMPK becomes activated independently of AXIN1, presumably via conformational changes in AMPK that allow LKB1 to directly phosphorylate Thr172. Importantly, we have found that AXIN2 (also known as AXIL or Conductin), which shows redundancy with AXIN1 in Wnt signaling, ${ }^{50}$ can also functionally replace AXIN1 in bridging LKB1 and AMPK. This spatiotemporal regulation of AMPK complexes in different compartments may be due to expression of different combinations of subunit isoforms and may have profound physiological and pharmacological implications.

\section{RESULTS}

Basal AMP is sufficient for activation of the lysosomal pool of AMPK

We previously showed that genetic knockout of either AXIN1 or LAMTOR1, critical components of the lysosomal AMPK activation complex, blocked AMPK activation (assessed by phosphorylation of Thr172) in mouse embryo fibroblasts (MEFs) deprived of glucose. ${ }^{18}$ We also reported that AMPK activation under these conditions was rapid (within $10 \mathrm{~min}$ ) and was not accompanied by any increases in cellular AMP:ATP or ADP:ATP ratios. ${ }^{14}$ However, we also found that more severe nutrient stress, caused by removal of both glucose and glutamine, caused a delayed activation of AMPK (up to $2 \mathrm{~h}$ ) that was independent of AXIN1 or LAMTOR1. ${ }^{14}$ These results suggested that glucose starvation may exclusively activate the lysosomal pool of AMPK, whereas complete nutrient withdrawal may activate non-lysosomal pools. To address this, we optimized methods to perform subcellular fractionation in MEFs (validation shown in Supplementary information, Fig. S1a-c) and systematically determined which pools of AMPK (cytosolic, lysosomal, mitochondrial, and nuclear) are activated by glucose deprivation. As shown in Fig. 1a and Supplementary information, Fig. S1d, Thr172 phosphorylation was only detected in the lysosomal fraction, but not in cytosolic, mitochondrial or nuclear fractions from MEFs cultured in low glucose ( $5 \mathrm{mM}$ or below), in which AMP:ATP and ADP:ATP ratios were unchanged (Fig. 1b). Similar results were obtained in HEK293T cells (Supplementary information, Fig. S1e, f). Subcellular fractionation of liver homogenates from mice starved for $16 \mathrm{~h}$, which showed no changes in adenine nucleotide ratios, also indicated that Thr172 phosphorylation was only detected in the lysosomal fraction (Fig. 1c, d). Interestingly, we found that under these conditions, phosphorylation of ACC1, but not ACC2, was increased (Fig. 1e, f; Supplementary information, Fig. S1g). Increased phosphorylation at the AMPK sites on SREBP1C, TSC2, Raptor and HDAC4, but not MFF, also occurred (Fig. 1g). These data suggest that inhibition of synthesis of lipid, protein and carbohydrates is an early event during the response to glucose starvation and occurs prior to any energy stress. In addition, phosphorylation of Ser660 of TBC1D1 involved in glucose uptake was also detected after glucose starvation, indicating an immediately early pro-catabolic activity (Fig. 1g).

It has been well established that AXIN2, which shares the RGS and DIX domains as well as the binding sites for $\beta$-catenin, GSK3, Diversin, and Smad3, is functionally equivalent to AXIN1, although AXIN2 is not expressed ubiquitously like AXIN1 but instead in tissue- and developmental-stage-specific patterns. ${ }^{50,51}$ In the course of testing for the generality of the mechanisms linking glucose sensing to AMPK activation in various cell lines, we observed that AMPK was still activated by glucose starvation in HEK293T cells even when AXIN1 was knocked down (Fig. 1h). However, AXIN2 was expressed at a readily detectable level in these cells, compared to MEFs, HEK293 cells, and the liver (Supplementary information, Fig. S1h, i), implying that in HEK293T cells AXIN2 was compensating for AXIN1 when the latter was depleted. Moreover, knockdown of AXIN1 in HEK293T cells elevated the levels of AXIN2 (Supplementary information, Fig. S1h). We also knocked down AXIN1 in AXIN2 ${ }^{-1-}$ HEK293T cells and found that the activation of AMPK by glucose starvation was indeed largely abrogated (Fig. 1h). Similarly, introduction of AXIN2 into AXIN1 $1^{-1-}$ MEFs (in which AXIN2 was almost undetectable) led to a significant activation of AMPK upon glucose starvation (Fig. 1i). In conclusion, our results support the functional equivalence of AXIN1 and AXIN2 in the lysosomal pathway of AMPK activation.

Modest increases in AMP activate cytosolic AMPK in an AXIN1dependent manner

Surprisingly, unlike MEFs or mouse liver, ${ }^{18}$ activation of AMPK by glucose starvation was still observed in HEK293 cells in which LAMTOR1 was knocked down (Fig. 2a). To reconcile these differences, we performed subcellular fractionation assays in HEK293 cells and found LAMTOR1-independent activation of both cytosolic and lysosomal AMPK, which was blocked when AXIN1 was knocked down (Fig. 2b). This evoked previous findings that there were modest increases in AMP/ATP and ADP/ATP ratios in HEK293 cells upon glucose removal, ${ }^{14}$ suggesting that AXIN1 can also act as a bridge that tethers AMPK and LKB1 in the cytosol 
a

Cytosol

GIc (mM) 251053100025105310

$\mathrm{G} \ln (4 \mathrm{mM})+++++$ +

IB: $\mathrm{p}$-AMPKa

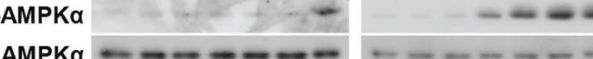

AMPKa $-\ldots-\ldots \ldots$
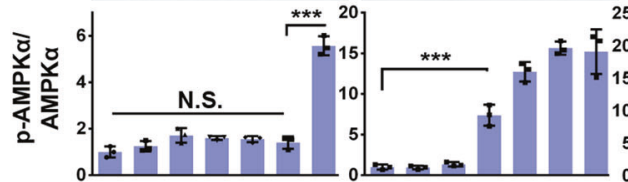

b

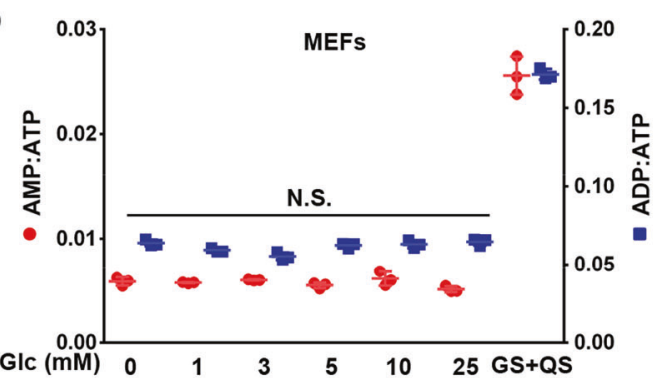

C

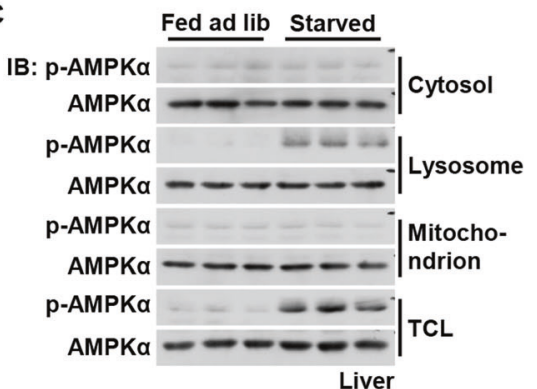

h

SiAXIN1 $\frac{\operatorname{AXIN2}^{+/+}}{-++}+\frac{\operatorname{AXIN2-1-}}{-++}$

GIc (mM) $25 \quad 0 \quad 250250250$

IB: p-AMPKa

AMPKa - - - - -

p-ACC $-\cdots$

$\mathrm{ACC}----\cdots$

AXIN1 - - -

AXIN2 - - -

Tubulin - - - - -

HEK293T

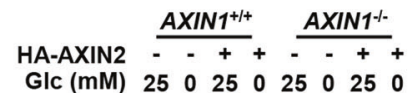

B: p-AMPKa - - -

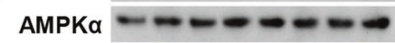

$\mathrm{p}-\mathrm{ACC}-6-0---6$

ACC $\ldots \ldots \ldots \ldots$

AXIN1 - - - -

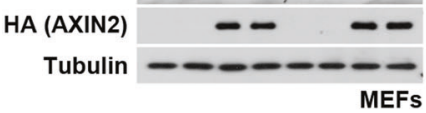

f
Mitochondrion

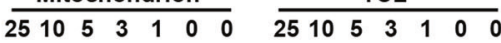

$++++++-++++++$

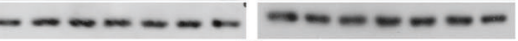

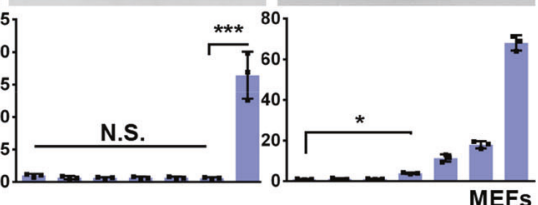

e

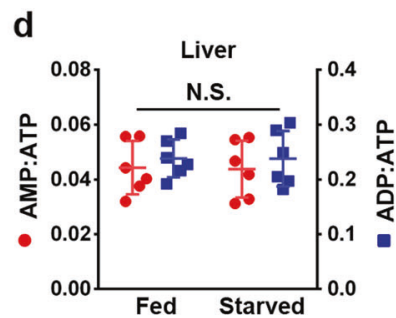

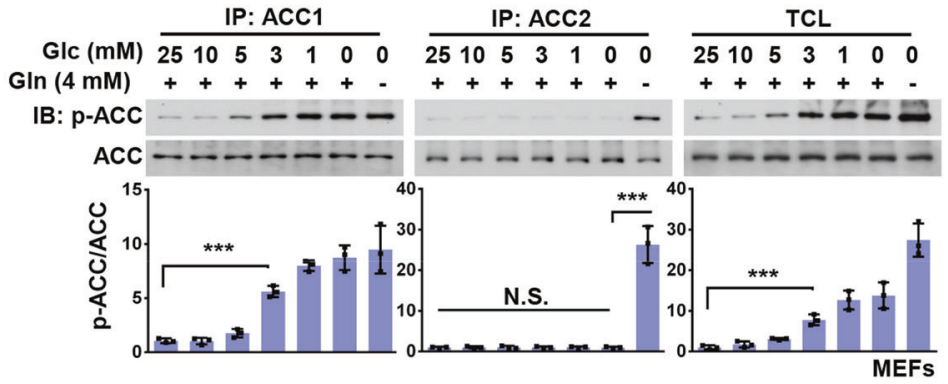
Fed ad lib Starved Fed ad lib Starved Fed ad lib Starved

IB: p-ACC

$\mathrm{ACC}-(--\cdots-\cdots-\cdots-1-$

Liver

g GS : + +

QS - - +

AICAR (2 mM) - - - +

IB: p-SREBP1 …

SREBP1 - - -

p-Raptor -6

Raptor - - -

p-TSC2 - -

$\mathrm{TSC} 2=0$

p-HDAC4

HDAC4 $=-0$

p-ULK1 $=--$

ULK1 $+k+t+1$

p-MFF $=-==$

MFF

p-Beclin-1

p-TBC1D1 - -

TBC1D1 -

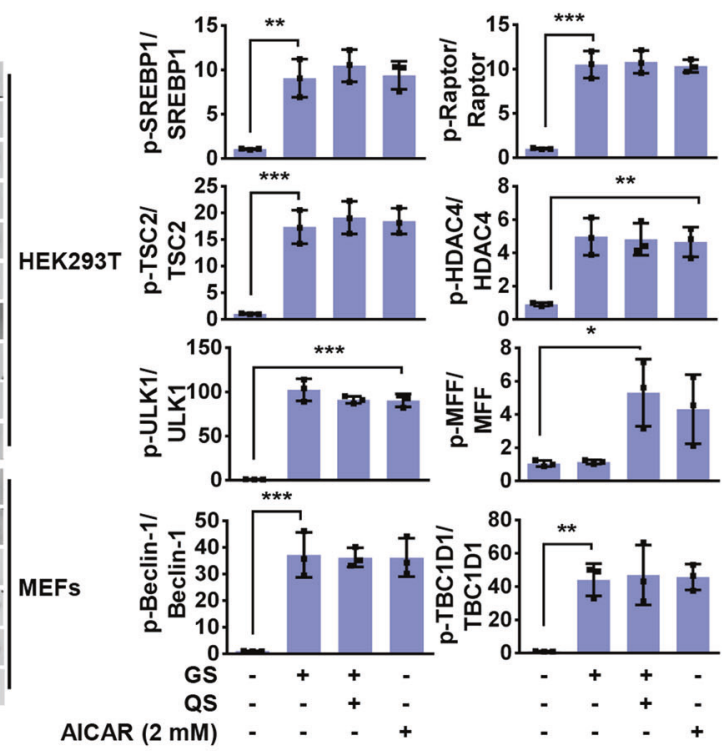

when AMP is modestly elevated. ${ }^{19}$ In this study, cellular AMP was increased in response to glucose removal from 30 to $60 \mu \mathrm{M}$, which was associated with an increase in AMP/ATP ratio from 0.006 to 0.015 (Fig. 2c), a similar extent to that observed in MEFs undergoing early phases of severe starvation. ${ }^{14}$ Under these conditions, AMPK phosphorylated ACC1 and ACC2 in a similar manner to that seen in MEFs upon glucose starvation (Fig. 2d; Supplementary information, Fig. S2a). We also used AICAR to mimic a moderate increase in AMP in MEFs. MEFs treated with $0.6 \mathrm{mM}$ AICAR yielded an estimated intracellular ZMP concentration of $1.2 \mathrm{mM}$ which, given that ZMP is about 40 -fold less potent than AMP for AMPK activation, ${ }^{52}$ is equivalent to around $50 \mu \mathrm{M}$ 
Fig. 1 Basal AMP Is Sufficient for Activation of the Lysosomal Pool of AMPK. a Low glucose exclusively activates the lysosomal pool of AMPK in MEFs. MEFs were grown in full medium and then switched to DMEM containing reduced concentrations of glucose for $2 \mathrm{~h}$, or to DMEM lacking both glucose and glutamine (starvation for glucose plus glutamine, GS + QS). Cytosolic, lysosomal, and mitochondrial fractions were prepared following the methods described in "Materials and Methods". Fractions were then subjected to analysis of p-AMPK $\alpha$ and p-ACC by immunoblotting using the indicated antibodies, followed by densitometry analysis. Statistical analysis results were shown in mean \pm SD; ${ }^{* * *} p<0.001,{ }^{*} p<0.05$, N.S., not significant by ANOVA, $n=3$. b AMP:ATP and ADP:ATP ratios are not changed in MEFs in low glucose. Adenylate nucleotide ratios in MEFs treated as in a were measured by CE-MS. Results are mean \pm SD; N.S., not significant by ANOVA, $n=3$. c Starvationinduced AMPK activation in liver takes place on lysosome. Mice were fed ad libitum or starved for $16 \mathrm{~h}$, followed by fractionation of cytosol, lysosomes and mitochondria from liver homogenates, and subsequent immunoblotting using the indicated antibodies. d AMP/ATP and ADP/ ATP ratios are unchanged in the liver of starved mice. Mice were fed or starved as in (c), freeze-clamped liver samples prepared, and AMP/ATP and ADP/ATP ratios measured by CE-MS. Results are mean \pm SD, $n=6$; N.S., not significant by Student's $t$-test. e, f ACC1, but not ACC2, is phosphorylated in MEFs starved for glucose or in the liver of starved mice. MEFs were glucose starved or severely starved as in a, while mice were fed or starved as in c. Endogenous ACC1 and ACC2 in MEFs (e) or mice livers (f) were individually immunoprecipitated, followed by immunoblotting. Statistical analysis data of experiments in e were shown in mean \pm SD; ${ }^{* * *} p<0.001$, N.S., not significant by ANOVA, $n=3$. g Phosphorylation of SREBP1c, TSC2, Raptor, HDAC4, ULK1, Beclin-1, and TBC1D1, but not MFF, is observed in low glucose. HEK293T cells or MEFs were incubated in DMEM medium with $(25 \mathrm{mM})$ or without glucose for $2 \mathrm{~h}$, followed by analysis of phosphorylation levels of AMPK substrates as indicated. Statistical analysis data were shown in mean \pm SD; ${ }^{*} p<0.05,{ }^{* *} p<0.01,{ }^{* * *} p<0.001$, by ANOVA, $n=3$. h AXIN1 and AXIN2 are functionally equivalent in the lysosomal pathway of AMPK activation in HEK293T cells. AXIN2 ${ }^{-1-}$ HEK293T cells and its wildtype control were infected with lentivirus expressing siRNA against AXIN1. Cells were starved for glucose for $2 \mathrm{~h}$ and then lyzed, followed by immunoblotting. i Re-introduction of AXIN2 into $A X I N 1^{-1}$ MEFs restores glucose starvation-induced AMPK activation. AXIN1 ${ }^{-1-}$ MEFs (and its wildtype control) were infected with lentivirus expressing HA-tagged AXIN2. Cells were then starved for glucose for $2 \mathrm{~h}$, followed by immunoblotting. Experiments in this figure were performed three times, except $\mathbf{d}$ and $\mathbf{i}$ twice. See also Supplementary information, Fig. S1

AMP (including $\sim 20 \mu \mathrm{M}$ endogenous AMP), a concentration similar to the estimate in HEK293 cells upon glucose starvation (Supplementary information, Fig. S2b). Consistently, we found an AXIN1-dependent, LAMTOR1-independent activation of both lysosomal and cytosolic AMPK, similar to that observed in HEK293 cells (Fig. 2e-h; Supplementary information, Fig. S2c, d). Taken together, these results suggest that AXIN1 also participates in the activation of cytosolic AMPK when AMP levels are moderately increased.

Further confirmation that AXIN1 can play a role in bridging LKB1 to AMPK in the activation of cytosolic AMPK as well as in the lysosomal pathway comes from our observation that in both lysosomal and cytosolic fractions, increased LKB1-AMPK interaction induced in conditions that caused elevation of AMP to moderate levels was dampened in the absence of AXIN1 (Fig. 2i). In vitro reconstitution experiments showed that addition of a moderate level of AMP $(60 \mu \mathrm{M})$ significantly promoted the phosphorylation of AMPK by LKB1 in vitro in an AXIN1dependent manner (Supplementary information, Fig. S2e). Taken together, these results demonstrate that after moderate elevation of AMP, AXIN1 alone can tether LKB1 to AMPK without the necessity for the $v$-ATPase-Ragulator complex involved in the lysosomal pathway.

Mitochondrion-associated ACC2 is phosphorylated by AMPK only after severe nutrient stress

We next analyzed the regulation of AMPK and its substrates under severe nutrient stress. When MEFs were severely starved or treated with $2 \mathrm{mM}$ AICAR (yielding $3.6 \mathrm{mM}$ intracellular ZMP, approximately equivalent to $110 \mu \mathrm{M}$ AMP, including approximately $20 \mu \mathrm{M}$ endogenous AMP) and thus mimicking the levels of AMP accumulated under severe nutrient stress, full activation of AMPK in the cytosolic, lysosomal and mitochondrial pools was observed (Fig. 3a, b). Such a full phosphorylation of AMPK under severe nutrient stress was also observed in HEK293 and HEK293T cells (Fig. 2a, b; Supplementary information, Fig. S1e). Of note, no nuclear AMPK activation could be detected under any level of stress (Supplementary information, Fig. S1d). Under these conditions, we found that ACC2 was phosphorylated as well as ACC1 (Fig. 3c). Compared with ACC1, ACC2 has an additional N-terminal hydrophobic sequence that specifically targets it to the mitochondrial outer membrane ${ }^{31}$ (Supplementary information, Fig. S3a-c). We thus probed the p-ACC signals in cytosolic and mitochondrial fractions and found that the mitochondrial p-ACC could only be detected under conditions of severe nutrient stress (Fig. 3d). To unequivocally determine that the $\mathrm{p}$-ACC signals seen after severe nutrient stress were contributed by $\mathrm{ACC} 2$, we generated $A C C 1^{-1-}$ and $A C C 2^{-1-}$ MEFs (Fig. S3d). Knockout of $A C C 2$, unlike that of $A C C 1$, had no effect on the phosphorylation of ACC upon glucose starvation or after a moderate elevation of AMP, but blocked the increase of the p-ACC signal in severe nutrient stress (Fig. 3e). Moreover, we found that levels of malonyl-CoA were decreased by $50 \%$ in MEFs starved for glucose only and were further decreased by up to $90 \%$ under severe nutrient starvation. This represents a similar reduction as that caused by treatment with TOFA (a panACC inhibitor) and occurs without any significant change on its precursor, acetyl-CoA (Fig. 3f). Consistently, both ACC1 and ACC2, along with AMPK from all the fractions, were phosphorylated in livers of mice subjected to hepatic ischemia, where a 22 -fold increase of AMP:ATP ratio was detected (equivalent to an increase of AMP from 20 to $475 \mu \mathrm{M}$, estimated as described previously, ${ }^{53}$ Fig. 3g, h; Supplementary information, Fig. S3e). A further decrease of malonyl-CoA, compared with that seen after 16-h starvation, was also observed (Fig. 3i). We found that when there were large increases in AMP, knockout of AXIN1 or LAMTOR1 did not block the phosphorylation of ACC1, ACC2 or AMPK in either the cytosolic, lysosomal or mitochondrial fractions (Supplementary information, Fig. S3f-i).

ACC1 and ACC2 contain very similar AMPK substrate recognition motifs ${ }^{54}$ (consequently phospho-specific antibodies recognize both) but vary in their subcellular locations. We hypothesized that the targets of AMPK may be regulated in a spatiotemporal manner under different stress conditions. To test this, we replaced the endogenous AMPK- $\beta$ subunits with modified forms that target the complex to specific locations. It is known that $\mathrm{N}$-myristoylation is required for AMPK association with intracellular membranes such as the lysosome ${ }^{14}$ and the mitochondrion. ${ }^{24}$ We generated two fusion constructs with modifications at the $\mathrm{N}$-terminus of the $\beta 1$ subunit: either adding LAMP2 (for tethering to the lysosomal surface, referred to as lyso- $\beta 1$ ) or TOMM20 (for tethering to mitochondrial outer membrane, referred to as mito- $\beta 1$ ) and also the $\beta 1-\mathrm{G} 2 \mathrm{~A}$ mutation (preventing $\mathrm{N}$-myristoylation, referred to as cyto- $\beta 1$ ) (Fig. 3j). Before reintroduction of the engineered constructs to HEK293T cells, we knocked out the $\beta$ subunits ( $\beta 1$ and $\beta 2$ ) of AMPK to generate AMPK $\beta$-DKO cells (validation in Supplementary information, Fig. S4a). Lyso- $\beta 1$, mito- $\beta 1$ and cyto- $\beta 1$ were then individually re-introduced to the AMPK $\beta-D K O$ cells. The engineered $\beta 1$ subunits assembled into heterotrimeric AMPK complexes as occurs with the wild-type AMPK- $\beta 1$, and became appropriately localized as validated by immunostaining 
a

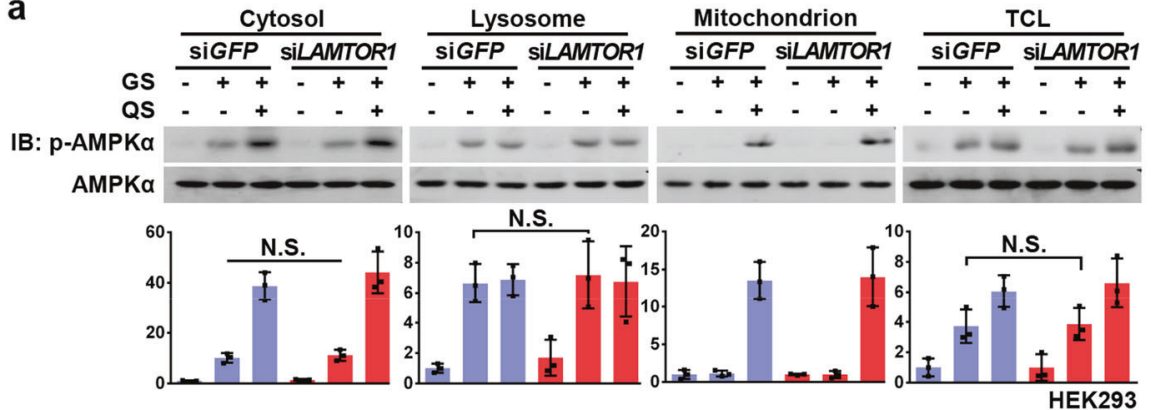

b

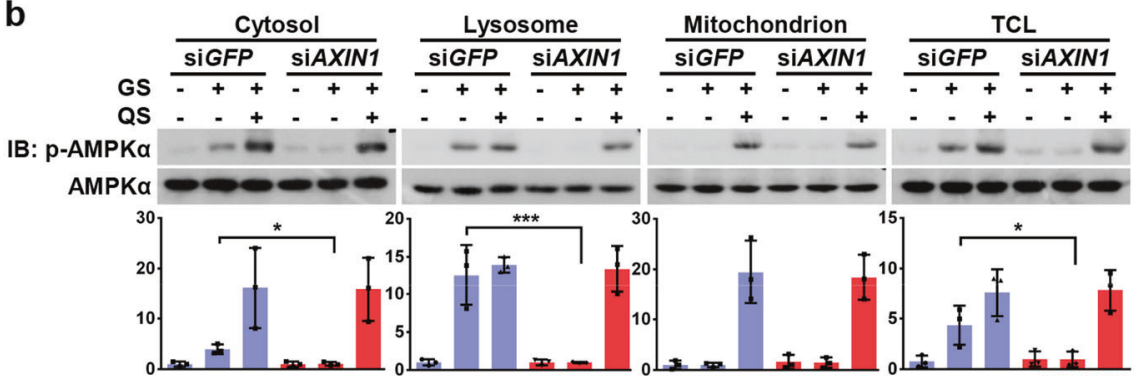

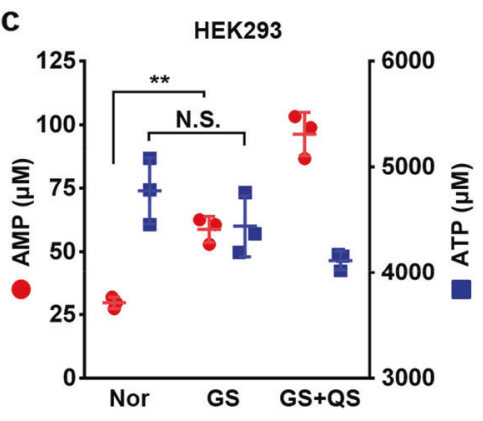

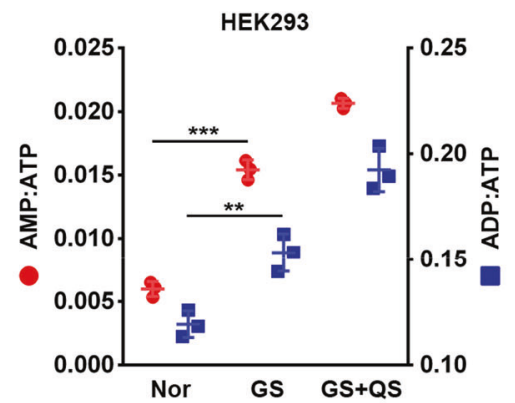

d

d
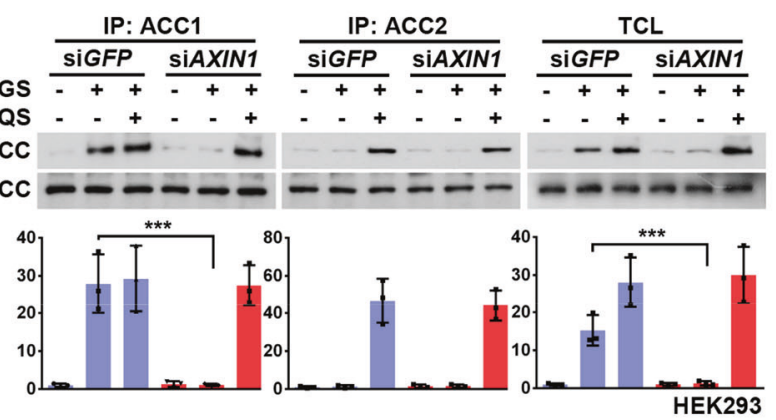

e

Cytosol Lysosome Mitochondrion TCL

GS - + + + + - + - + + + + +

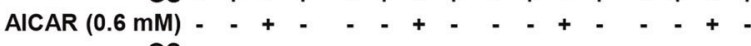

IB: $\mathrm{p}-\mathrm{AMPKa}$

AMPKa

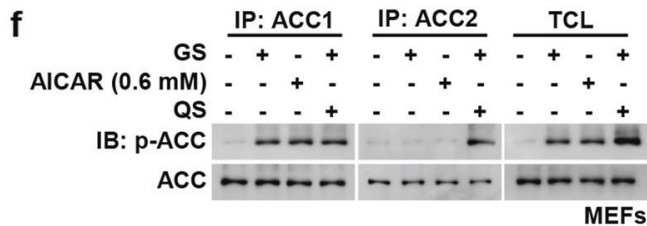

MEFs

g

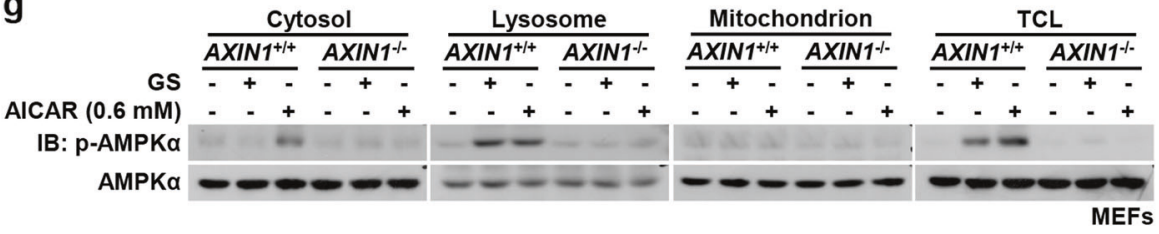

i AXIN1 ${ }^{+++} A X I N 1^{1+}$

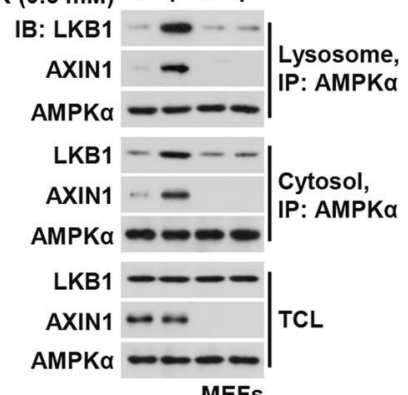

h

Cytosol Lysosome Mitochondrion TCL

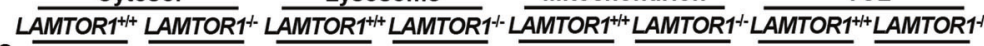

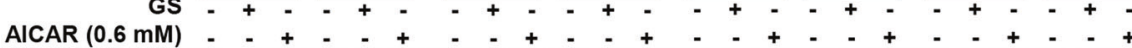

IB: p-AMPKa $\quad-\quad-$

AMPKa $-0-0-1-\cdots-1-0-1-0$
AMPK substrate, ${ }^{47}$ was phosphorylated in a similar manner to ACC2 (Fig. 1g).

Roles of AMP in the hierarchical activation of AMPK

We postulated that upon starvation for glucose only, basal AMP might act as a necessary cofactor for AMPK activation via the lysosomal pathway, whereas elevated AMP may bind to additional sites on AMPK. Indeed, when the AMPK- $\gamma 1$ mutant D317A, which affects the "non-exchangeable" site for AMP (site 4), was reintroduced into HEK293T cells with knockouts of all $\gamma$ subunits $(\gamma 1$ to $\gamma 3$ ) (AMPKY-TKO cells, Supplementary information, Fig. S4e), the activation of AMPK upon glucose starvation was severely 
Fig. 2 Modest Increases in AMP Activate Cytosolic AMPK in an AXIN1-dependent Manner. a Glucose starvation activates both lysosomal and cytosolic AMPK in a LAMTOR1-independent manner in HEK293 cells. HEK293 cells were infected with siRNA against LAMTOR1, and were deprived of glucose or both glucose and glutamine for $2 \mathrm{~h}$, followed by fractionation and immunoblotting. Statistical analysis data were shown on lower panel, in mean \pm SD; N.S., not significant by ANOVA, $n=3$. b AXIN is required for glucose starvation-induced, cytosolic AMPK activation in HEK293 cells. Cells were infected with siRNA against AXIN1, followed by glucose starvation or severe nutrient starvation as in a. P-AMPK $\alpha$ level was then determined by immunoblotting, followed by densitometry analysis, and the data were shown in mean \pm SD; *** $p<$ $0.001,{ }^{*} p<0.05$ by ANOVA, $n=3$. c The AMP levels and AMP/ATP and ADP/ATP ratios are modestly increased in HEK293 cells, unlike MEFs, after glucose starvation. HEK293 cells were regularly cultured (Nor), starved for glucose for $2 \mathrm{~h}$ (GS), or starved for both glucose and glutamine for $2 \mathrm{~h}(\mathrm{GS}+\mathrm{QS}$ ), followed by determination of concentrations (upper panel) and ratios (lower panel) of adenylate nucleotide by CE-MS. Results are mean $\pm S D ;{ }^{* *} p<0.01,{ }^{* *} p<0.001$ by ANOVA, $n=3$. d ACC1, but not ACC2, is phosphorylated in HEK293 cells after glucose starvation. Cells were starved as in $\mathbf{b}$, in which endogenous ACC1 and ACC2 were individually immunoprecipitated and analyzed by immunoblotting. Statistical analysis data were shown in mean \pm SD; ${ }^{* * *} p<0.001$ by ANOVA, $n=3$. e AICAR at low concentrations activates cytosolic AMPK as does moderately increased AMP. MEFs were treated with $0.6 \mathrm{mM}$ AICAR for $2 \mathrm{~h}$, or starved for glucose or both glucose and glutamine for $2 \mathrm{~h}$, followed by fractionation and immunoblotting. $\mathbf{f} A C C 1$, but not ACC2, is phosphorylated in MEFs treated with low concentrations of AICAR. MEFs were treated with AICAR or severely starved as in e. The endogenous ACC1 and ACC2 were then individually immunoprecipitated and analyzed by immunoblotting. $\mathbf{g}$, h AICAR at low concentrations activates cytosolic AMPK in an AXIN1-dependent, LAMTOR1-independent

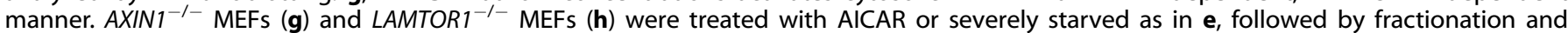
immunoblotting. $\mathbf{i}$ Knockout of AXIN1 blocked the association between LKB1 and AMPK under moderate AMP in MEFs. Cells were treated with $0.6 \mathrm{mM}$ AICAR for $2 \mathrm{~h}$, followed by fractionation. Endogenous AMPK $\alpha$ from lysosomal and cytosolic fractions was then immunoprecipitated, and its interaction with AXIN1 and LKB1 was analyzed by immunoblotting with indicated antibodies. Experiments in a, b, d, $\mathbf{g}, \mathbf{h}$, and $\mathbf{i}$ were performed three times, and the others twice. See also Supplementary information, Fig. S2

dampened (Fig. 4a). This is consistent with our previous finding that the AMP-promoted interaction between LKB1 and AMPK is impaired by this mutant. ${ }^{19}$ We also introduced the R531G mutant, in which the exchangeable site for AMP (site 3$)^{8}$ is disrupted, into AMPKY-TKO cells, and found that the phosphorylation of lysosomal AMPK upon glucose starvation remained unaffected. This contrasted to cytosolic and mitochondrial AMPK phosphorylation which was blocked under moderate and high AMP levels (Fig. 4b). Similarly, phosphorylation of ACC2 was blocked in R531G-expressing cells (Fig. 4c). It is not clear why the R531Gcontaining AMPK complex can respond to glucose starvation, yet fails to be activated by severe starvation (Fig. 4b, c). It is possible that under severe starvation such a mutation perturbs the overall structure of the AMPK complex, thereby blocking its activation by any mode. We also determined whether AMP levels underlie the activation of cytosolic and mitochondrial AMPK by manipulating the generation of AMP by knocking out adenylate kinase 1 (AK1, which catalyzes the conversion of 2 ADP to 1 ATP and 1 AMP) in MEFs (Supplementary information, Fig. S4f). Indeed, we found that knockout of $A K 1$ significantly dampened the activation of mitochondrial- and cytosolic-localized AMPK, and the phosphorylation of ACC2 in conditions of severe nutrient starvation (Fig. 4d; Supplementary information, Fig. S4g).

\section{DISCUSSION}

In this study, we have systemically determined how differentially localized AMPK complexes are regulated to affect distinct target phosphorylation in response to different stresses (Fig. 4e). Under conditions of glucose starvation that did not elevate cellular AMP: ATP or ADP:ATP ratios, only lysosomally localized AMPK was activated and exclusively through the lysosomal pathway. However, when AMP was moderately elevated, cytosolic AMPK was also activated in addition to lysosomal AMPK. Activation induced by moderate increases in AMP was not mediated via the lysosomal pathway, because knockout of LAMTOR1 (a subunit of the Ragulator complex) had no effect although activation was still dependent on AXIN1. In support of this, moderately elevated AMP promoted the formation of a complex between AXIN1, LKB1 and AMPK. Interestingly, mitochondrial AMPK was not activated by moderate increases in AMP, but only in response to more severe nutrient stress when there were larger increases in AMP. One possible explanation for this is that the concentrations of ATP are higher in the vicinity of mitochondria, inhibiting the activation of AMPK by AMP. However, when cellular AMP is elevated to higher levels during severe nutrient stress or ischemia, mitochondrial
AMPK becomes activated independently of AXIN1, phosphorylating the mitochondrially localized substrates, ACC2 and MFF.

Basal AMP is believed to be maintained at low levels by the freely reversible adenylate kinase reaction (2ADP $\leftrightarrow$ ATP + AMP), with the high ATP:ADP ratio in unstressed cells driving the reaction towards ADP and away from AMP. AMP is thought to be constantly bound as a cofactor to site 4 of the AMPK- $\gamma$ subunit, ${ }^{7,8}$ perhaps rendering the AMPK complex ready for activation through the aldolase-v-ATPase-Ragulator pathway when glucose runs low. Although AXIN is required for activation of both lysosomal and cytosolic AMPK, increases in AMP are required for activation of cytosolic AMPK. A possible explanation for this is that the presence of Ragulator and v-ATPase on the lysosome alters the conformation of the intrinsically disordered protein AXIN that can form various intramolecular loops ${ }^{56,57}$ such that it is more accessible to AMPK even at basal levels of AMP. By contrast, AXIN does not come into contact with the Ragulator:v-ATPase complex in the cytosol, so that only when AMP is bound at a site additional to site 4 (most likely site 3 , supported by the results obtained using the R531G mutant) which likely causes conformational changes therein, ${ }^{58,59}$ AXIN and LKB1 form a complex with cytosolic AMPK. Finally, when cells are subjected to severe nutrient stress, AMP is increased to such high levels that AMPK is fully occupied by AMP, and LKB1 does not require AXIN to bind AMPK.

AMPK is composed of three subunits, each occurring as more than one isoform $(\alpha 1, \alpha 2 ; \beta 1, \beta 2 ; \gamma 1, \gamma 2, \gamma 3)$. These isoforms, some of which are expressed in a tissue-specific manner, can form up to twelve different heterotrimeric combinations. ${ }^{60}$ It has been proposed that these different isoform combinations target AMPK to different subcellular locations. ${ }^{3,61,62}$ Indeed, studies have shown that isoform-specific activations of AMPK lead to different effects on metabolic controls. For example, compared to AICAR, A-769662 (the $\beta 1$-specific activator) only mildly stimulated fatty acid oxidation, although it robustly inhibited fatty acid synthesis. ${ }^{63}$ The AMPK complex containing the $\gamma 1$ D317A mutant was mainly cytosolic ${ }^{64}$ and failed to stimulate fatty acid oxidation despite its chronic activity. ${ }^{65}$ Based on the results in this paper, it is conceivable that different thresholds of stress are involved in triggering differentially localized AMPK heterotrimers and that different heterotrimeric AMPK complexes might have specificity for targets due to different subcellular locations. Indeed, it is already known that AMPK complexes containing $\gamma 1, \gamma 2$ and $\gamma^{3}$ differ in their sensitivity to activation by AMP and ADP.9

Our current study has shown that an immediate role of AMPK activation is to phosphorylate and inhibit factors involved in 
a

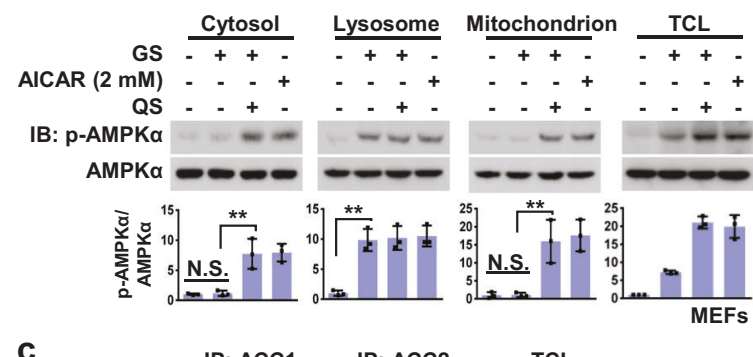

b

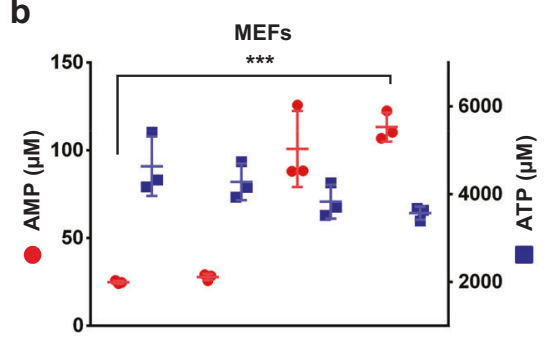

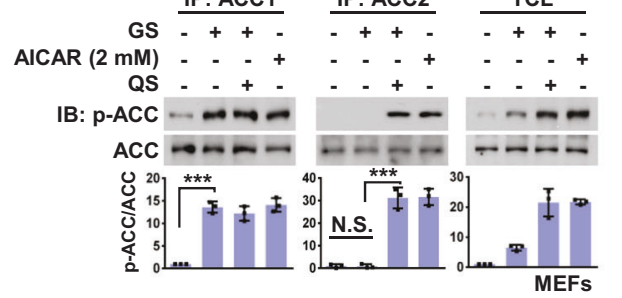

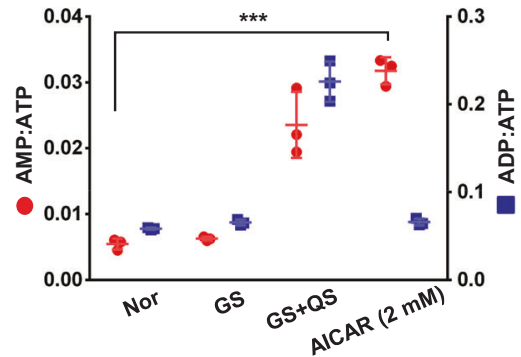

d

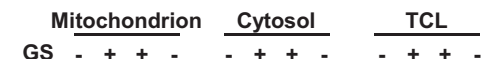

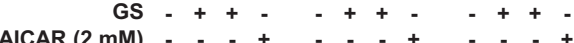

$-e^{-1+}$

IB: p-ACC $\quad-\infty-\infty-\infty$

ACC $\cdots \cdots \cdots+\cdots \cdots$

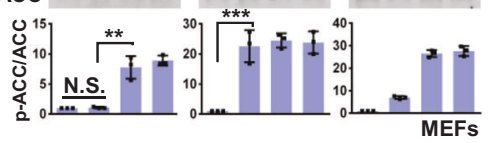

e

$\operatorname{ACC1}^{--} \quad \operatorname{ACC2}^{---}$

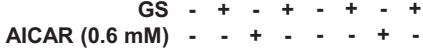

QS - - - + - - +

IB: $p-A C C$

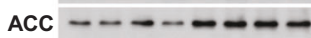

MEFs

g

Cytosol Lysosome Sham Ischemia

$\frac{\text { Lysosome }}{\text { Sham Ischemia }}$

Mitochondrion

TCL

Ischemia

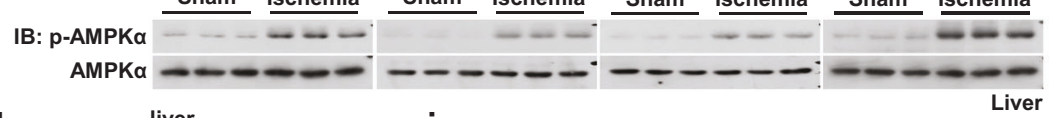

h

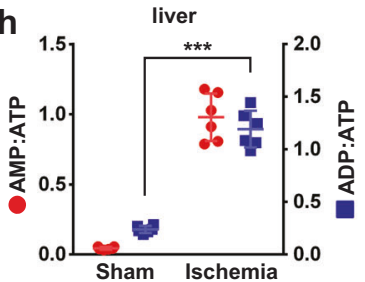

i
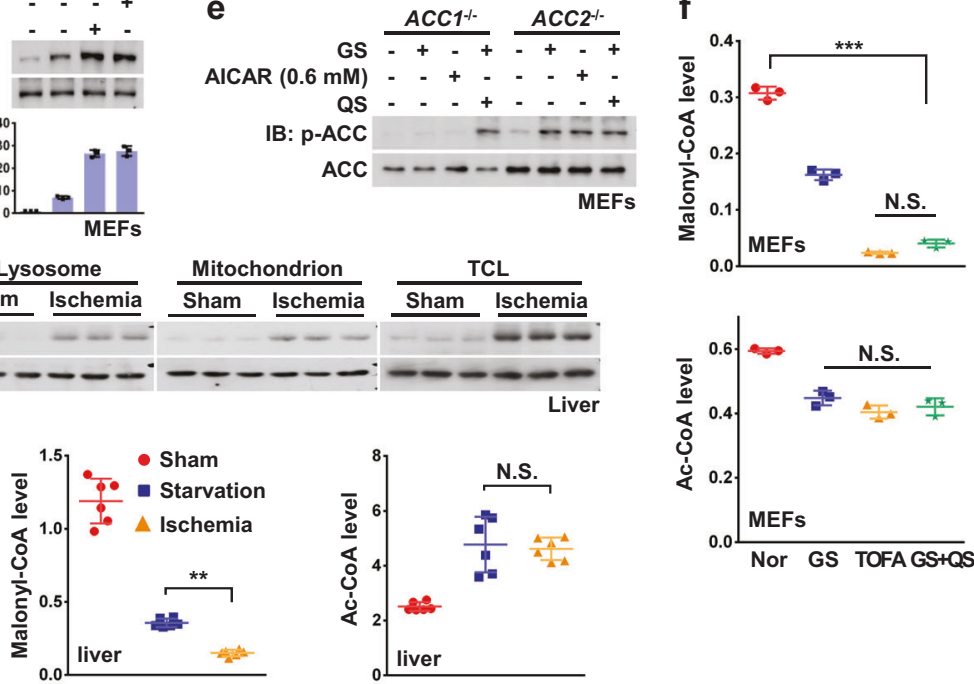

j

\begin{tabular}{|l|l|l|}
\hline LAMP2 & AMPKB1 & HA \\
\hline \multicolumn{2}{|c|}{ G2A } \\
Lyso- $\beta 1$
\end{tabular}
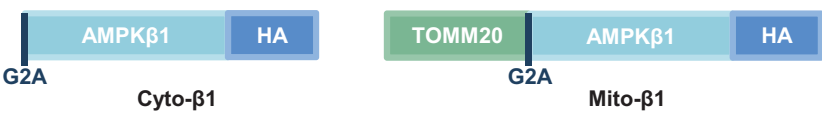

k

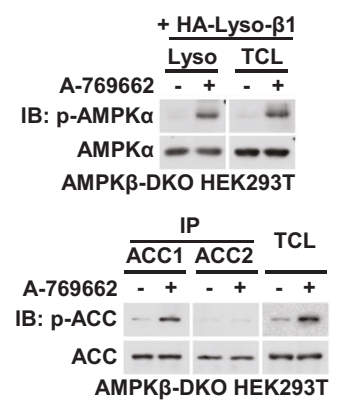

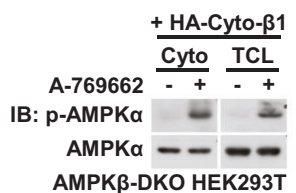

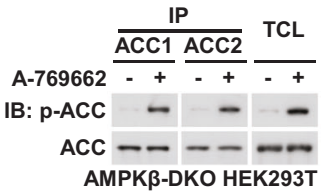

HA

(n)

AMPK $\beta$-DKO HEK293T

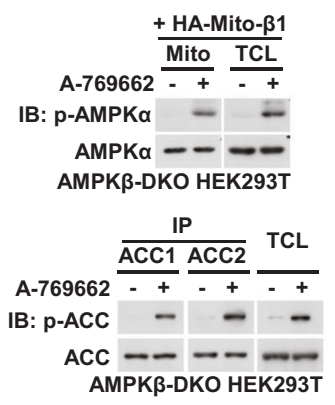

anabolic pathways including ACC1, SREBP1C, HDAC4, TSC2, and Raptor. It is interesting that ACC2 is phosphorylated only under conditions of more severe nutrient stress and ischemia, when there are larger increases in AMP. This indicates that fatty acid oxidation, a catabolic pathway, is increased during more severe stress, while fatty acid synthesis is shut down during a mild stress, i.e. lack of glucose. Since glucose is a major precursor for fatty acid synthesis in many cell types, it might make sense for this pathway to be switched off when there is reduced availability of glucose. By contrast, ACC2 appears to be phosphorylated only under 
Fig. 3 The Mitochondria-localized ACC2 Is Phosphorylated by AMPK Only after Severe Nutrient Stress. a Severe nutrient stress causes a full activation of AMPK in the cytosolic, lysosomal and mitochondrial pools. MEFs were starved for both glucose and glutamine for $2 \mathrm{~h}$, treated with $2 \mathrm{mM}$ AICAR for $2 \mathrm{~h}$, or starved for glucose for $2 \mathrm{~h}$ as a control, followed by fractionation and immunoblotting for $\mathrm{p}$-AMPK $\alpha$. Statistical analysis data were shown in mean $\pm \mathrm{SD} ;{ }^{* *} p<0.01$, N.S., not significant by ANOVA, $n=3$. b The AMP levels, and ratios of AMP/ATP and ADP/ ATP in MEFs under severe nutrient stress. Cells were treated with $2 \mathrm{mM}$ AICAR or starved for glucose as in a, in which adenylate concentrations (upper panel) and nucleotide ratios (lower panel) were measured by CE-MS. Note that in the group of AICAR treatment, the total levels of AMP were composed of the amount of ZMP-converted AMP (estimated according to a previous study ${ }^{52}$ ) and that of endogenous AMP. Results are mean \pm SD; ${ }^{* * *} p<0.001$ by ANOVA, $n=3$. c Both ACC1 and ACC2 are phosphorylated in MEFs under severe nutrient stress. Cells were treated as in a. The endogenous ACC1 and ACC2 were then individually immunoprecipitated and analyzed by immunoblotting. Statistical analysis data were shown in mean $\pm S D ;{ }^{* *} p<0.001$, N.S., not significant by ANOVA, $n=3$. d ACC1/2 in both cytosolic and mitochondrial pools can be phosphorylated in MEFs under severe nutrient stress. Cells were treated with AICAR or starved for glucose as in a, followed by fractionation and immunoblotting for p-ACC. Statistical analysis data were shown in mean \pm SD; ${ }^{* * *} p<0.001,{ }^{* *} p<0.01$, N.S., not significant by ANOVA, $n=$ 3. e ACC2 can only be phosphorylated in MEFs under severe nutrient stress. $A C C 1^{-1-}$ and $A C C 2^{-1-}$ MEFs were starved for glucose for $2 \mathrm{~h}$, treated with $0.6 \mathrm{mM}$ AICAR for $2 \mathrm{~h}$, or starved for both glucose and glutamine for $2 \mathrm{~h}$, followed by immunoblotting. $\mathbf{f}$ Levels of acetyl-coA and malonyl-CoA, the substrate and product respectively of ACC, in MEFs under different kinds of stress. Cells were starved or treated with AICAR as in a, except that $20 \mu \mathrm{M}$ TOFA (2-h-treatment) was used as an additional control, followed by determining acetyl-coA and malonyl-CoA levels by HPLC-MS. Results are mean \pm SD; ${ }^{* * *} p<0.001$, N.S., not significant by ANOVA, $n=3$. g All pools of AMPK, in the cytosol, lysosome and mitochondria can be activated in liver of mice subjected to hepatic ischemia. Mice were anesthetized. The left lateral and median lobes of liver were deprived of blood flow for $10 \mathrm{~min}$ by cross-clamping the hepatic artery and portal vein (described in Materials and Methods). Liver homogenates were then subjected to fractionation as in Fig. 1c, followed by immunoblotting. $\mathbf{h}$, i AMP/ATP and ADP/ATP ratios, acetyl-coA and malonyl-coA levels in livers from mice under starvation or hepatic ischemia. Mice were starved for $16 \mathrm{~h}$ or subjected to hepatic ischemia (for $10 \mathrm{~min}$ ), followed by measurement of AMP/ATP and ADP/ATP ratios by CE-MS (h) or acetyl-coA and malonyl-coA levels by HPLC-MS (i). Results are mean $\pm \mathrm{SD} ;{ }^{* * *} p<0.001$ by Student's $t$-test (h), ${ }^{* *} p<0.01$, N.S., not significant by ANOVA (i), $n=6$. j A schematic diagram showing the three fusion constructs of the $\beta 1$ subunit (with modifications at the N-terminus) that allow AMPK to locate on lysosomal surface, mitochondrial outer membrane, or in cytosol. k ACC2 can only be phosphorylated by cytosol-localized and the mitochondrion-localized AMPK. AMPK $\beta$-DKO HEK293T cells were infected with HA-tagged lyso- $\beta 1$ (left panel), cyto- $\beta 1$ (middle panel), and mito- $\beta 1$ (right panel),

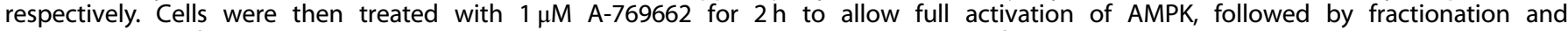
immunoblotting for analyzing p-AMPK $\alpha$, or by immunoprecipitation and immunoblotting for analyzing p-ACC1 and p-ACC2. Experiments in a, c, $\mathbf{d}, \mathbf{e}, \mathbf{g}$, and $\mathbf{k}$ were performed three times, and the others twice. See also Supplementary information, Figs. S3, S4

conditions of more severe nutrient stress (lack of both glucose and glutamine), when fatty acid oxidation may need to be facilitated as an alternate energy source. It is also interesting to note that ULK1 and Beclin-1 become phosphorylated at an early stage of glucose starvation, ${ }^{44}$ exactly the time point that AMPK is activated $^{14}$ although autophagy takes place only at a later stage in which additional factors such as accumulated ammonia, and the increased acetylation of VPS34 are involved in the formation of autophagosomes. ${ }^{66,67}$ Consistently, we also showed that the mitochondrial fission factor MFF, which promotes the formation of fragmented mitochondria prior to mitophagy/autophagy is only phosphorylated after severe starvation. Taken together, differently localized AMPK pools are differentially regulated, with AMPdependent and AMP-independent pathways operating in a spatiotemporal manner. Our paper supports the view that glucose sensing by the lysosomal AMPK activation pathway serves to act as a surveillance system monitoring the availability of glucose and switching off anabolic pathways, many of which require glucose for the provision of precursors. We also suggest that pharmacological activation of all pools of AMPK might have adverse effects on cellular and tissue physiology, and that new strategies may be required to activate the appropriate spatial and temporal pools of AMPK in order to develop effective and safe drugs for treating metabolic diseases.

Although we have demonstrated that different severities of nutritional stress modulate different pools of AMPK and different downstream targets, contingent on different cellular levels of $\mathrm{AMP}$, in some cases it remains unclear how this relates to the physiological roles of the targets. For example, we found that ACC2 is phosphorylated only after severe nutrient stress in MEFs and is not phosphorylated in liver even in mice starved overnight. However, fatty acid oxidation has been reported to be enhanced in mice that were starved overnight. ${ }^{68}$ Thus, elevated fatty acid oxidation under mild starvation may be caused by processes other than ACC2 phosphorylation. Indeed, it has been reported that increased circulating fatty acid, released primarily by adipose tissues, ${ }^{69}$ and enhanced expression of CPT1, ${ }^{70}$ may be involved under these circumstances. Another interesting issue is that ULK1 and Beclin-1 are among the substrates that are phosphorylated by
AMPK immediately after glucose starvation, yet autophagy does not take place upon short-term glucose starvation. It therefore remains unclear what the roles of the phosphorylation of ULK1 and Beclin- 1 by AMPK exert beyond autophagy, although ULK1/2 have been shown to play a role in modulating glucose metabolic fluxes, independently of autophagy. ${ }^{71}$

\section{MATERIALS AND METHODS}

\section{Antibodies}

Rabbit polyclonal antibody against LAMTOR1 was raised and validated as described previously, ${ }^{18}$ and was diluted 1:100 for immunoprecipitation (IP) or 1:500 for immunoblotting (IB). Rabbit anti-phospho-AMPKa-T172 (cat. \#2535, 1:1000 for IB), anti-AMPKa (cat. \#2532, 1:1000 for IB), anti-phospho-ACC-Ser79 (cat. \#3661, 1:1000 for IB), anti-ACC (cat. \#3662, 1:1000 for IB), anti-LKB1 (cat. \#3047, 1:1000 for IB), anti-AMPKB1/2 (cat. \#4150, 1:1000 for IB), anti-AMPKY1 (cat. \#4187, 1:1000 for IB), anti-AMPKy2 (cat. \#2536, 1:1000 for IB), anti-AMPKץ3 (cat. \#2550, 1:1000 for IB), anti-AXIN1 (cat. \#2074, 1:1000 for IB), anti-AXIN2 (cat. \#2151, 1:1000 for IB), anti-ACC1 (cat. \#4190, 1:1000 for IB and 1:100 for IP), anti-ACC2 (cat. \#8578, 1:1000 for IB and 1:25 for IP), anti-phospho-SREBP1cS372 (cat. \#9874, 1:500 for IB), anti-phospho-Raptor-S792 (cat. \#2083, 1:1000 for IB), anti-Raptor (cat. \#2280, 1:1000 for IB), antiphospho-TSC2-S1387 (cat. \#2280, 1:1000 for IB), anti-TSC2 (cat. \#4308, 1:1000 for IB), anti-phospho-HDAC4-S246 (cat. \#3443, 1:1000 for IB), anti-HDAC4 (cat. \#7628, 1:1000 for IB), antiphospho-MFF-S146 (cat. \#49281, 1:500 for IB), anti-MFF (cat. \#86668, 1:1000 for IB), andi-phospho-TBC1D1-S660 (cat. \#6928, 1:500 for IB), anti-TBC1D1 (cat. \#4629, 1:1000 for IB), anti-phosphoULK1-S555 (cat. \#5869, 1:1000 for IB), anti-phospho-Beclin-1-S93 (cat. \#14717, 1:1000 for IB), anti-Beclin-1 (cat. \#3495, 1:1000 for IB), anti-COXIV (cat. \#4850, 1:1000 for IB and 1:200 for IF), anti-PDI (cat. $\# 3501,1: 1000$ for IB), anti-Lamin B1 (cat. \#13435, 1:1000 for IB), anti- $\beta$-tubulin (cat. \#2128, 1:1000 for IB), anti-HA-tag (cat. \#3724, 1:200 for IF), mouse anti-Myc-tag (cat. \#2276, 1:1000 for IB), and HRP-conjugated mouse anti-rabbit lgG (conformation specific, cat. \#5127， 1:2000 for IB) antibodies were purchased from Cell Signaling Technology. Rabbit anti-AK1 (cat. 14978-1-AP, 1:500 

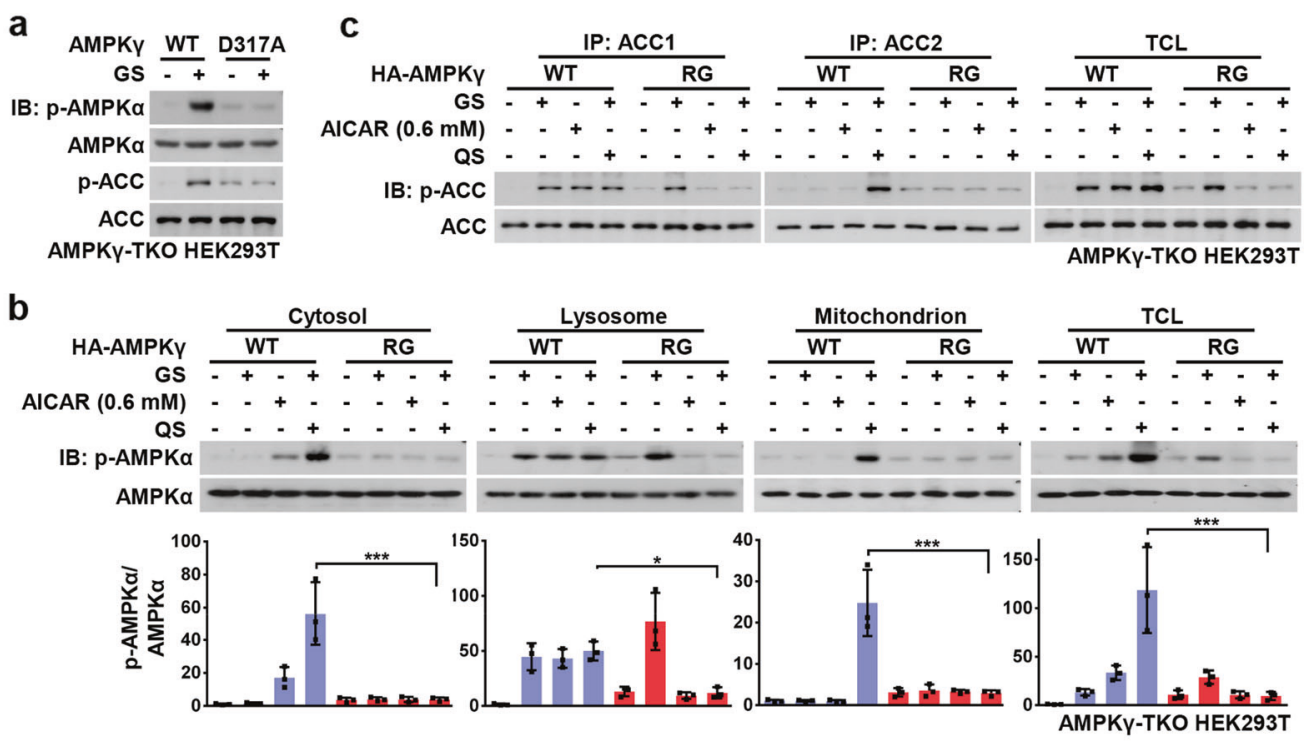

d

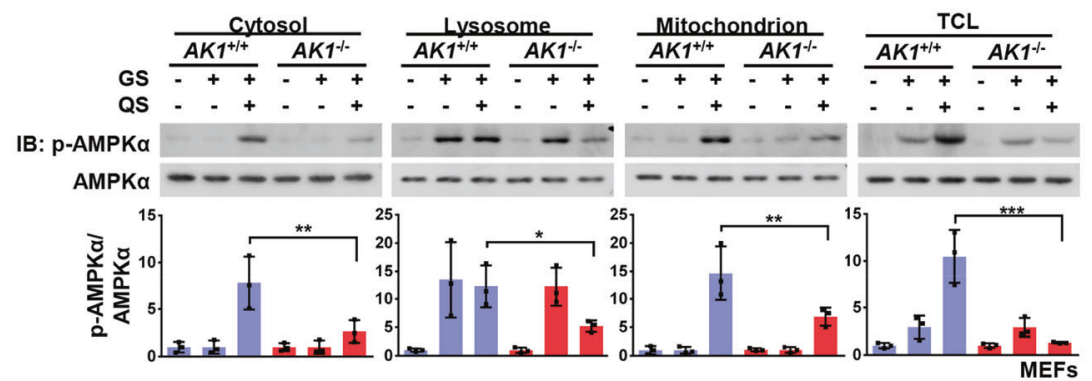

e

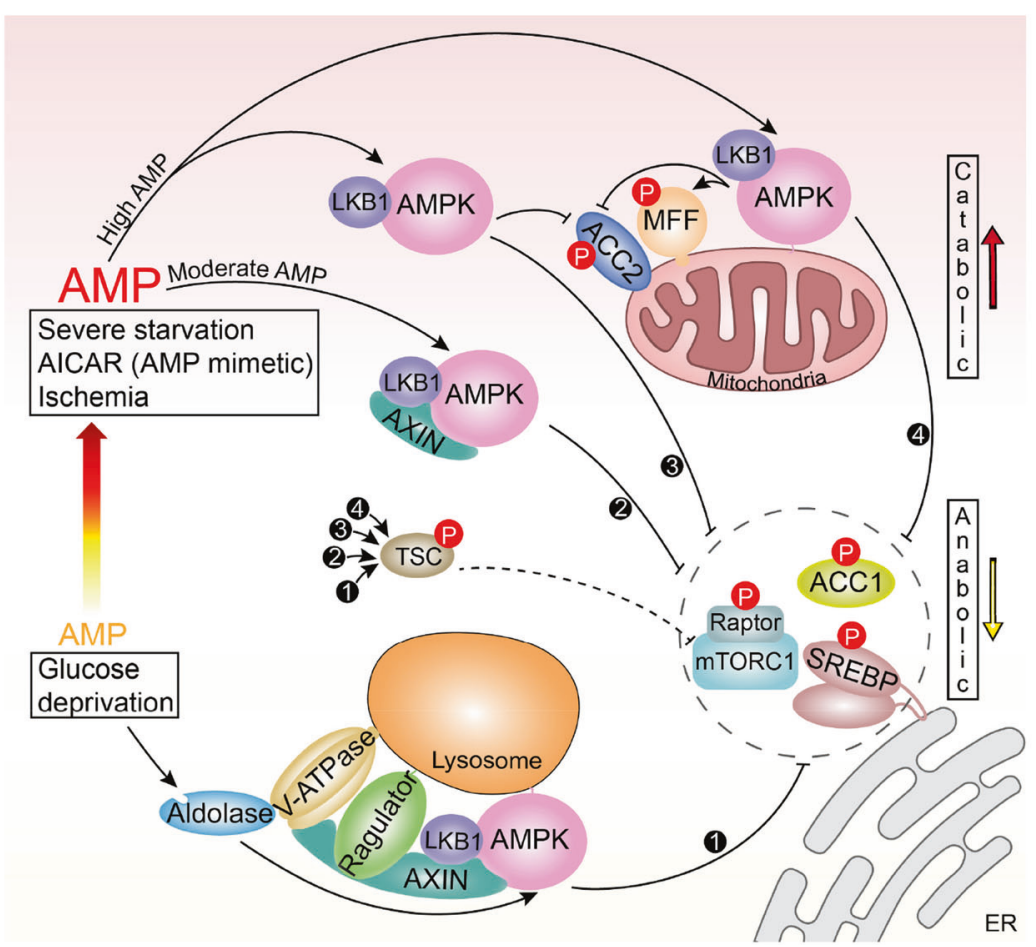

for IB) and rabbit anti AXIN2 (cat. 20540-1-AP, 1:1000 for IB, only for Supplementary information, Fig. S1h) were purchased from Proteintech. Mouse anti-LAMP1 (cat. ab13523, 1:200 for IF), antiAMPKa (cat. ab80039, 1:100 for IP) and rat anti-LAMP2 (cat. ab13524, 1:1000 for IB) were purchased from Abcam. The ANTI-
FLAG ${ }^{\circledR}$ M2 Affinity Gel (cat. A2220) and rabbit anti-ULK1 (cat. A7481, 1:1000 for IB) were purchased from Sigma. Goat anti-AXIN (cat. Sc-8567, 1:100 for IP and 1:60 for IF), rabbit anti-SREBP1 (cat. sc-366, 1:1000 for IB), and mouse anti-HA (cat. sc-7392, 1:2000 for IB and 1:200 for IF) antibodies were purchased from Santa Cruz 
Fig. 4 Roles of AMP in the Hierarchical Activation of AMPK. a AMPK $\gamma 1-D 317 A$ impairs glucose-starvation-induced AMPK activation. HA-tagged AMPK- $\gamma 1$ and its D317A mutant were re-introduced into AMPK $\gamma$-TKO HEK293T cells. Cells were then deprived of glucose for $2 \mathrm{~h}$, followed by immunoblotting. b, c AMPK $\gamma 2$-R531G blocks the phosphorylation of cytosolic and mitochondrial AMPK under moderate and high AMP levels. HA-tagged AMPK- $\gamma 2$ and its R531G mutant were re-introduced into AMPK $\gamma$-TKO HEK293T cells. Cells were then deprived of glucose for $2 \mathrm{~h}$, followed by fractionation and immunoblotting for analyzing p-AMPK $\alpha$ (b), or by immunoprecipitation and immunoblotting for analyzing $\mathrm{p}$ ACC1 and p-ACC2 (c). Statistical analysis data were shown in mean \pm SD; ${ }^{* * *} p<0.001,{ }^{*} p<0.05$, N.S., not significant by ANOVA, $n=3$. d Knockout of $A K 1$ significantly dampens the activation of mitochondrial- and cytosolic-localized AMPK in high AMP conditions. MEFs with $A K 1$ being knocked out were deprived of glucose or both glucose and glutamine for $2 \mathrm{~h}$, followed by fractionation and immunoblotting for analyzing $\mathrm{p}-\mathrm{AMPK} \alpha$. Statistical analysis data were shown in mean $\pm \mathrm{SD} ;{ }^{* * *} p<0.001,{ }^{* *} p<0.01,{ }^{*} p<0.05$, N.S., not significant by ANOA, $n=3$. e A simplified model depicting that the differentially compartmentalized pools of AMPK are activated with different dependencies on AXIN, and the severities of nutrient or energy stress. Glucose starvation, without increase of AMP levels, exclusively activates the lysosomal pool of AMPK through the AXIN-based pathway (1) which phosphorylates substrates including ACC1, SREBP1, TSC2, Raptor, HDAC4, ULK1, and TBC1D1 to elicit early anti-anabolic roles. Moderately increased AMP levels, during the early phase of severe starvation or after treatment of low concentrations of AICAR, activates cytosolic AMPK, in addition to the lysosomal AMPK (2), still dependent on AXIN. When AMP levels go up further as a result of severe starvation, ischemia, or treatment of high concentrations of AICAR, cytosolic AMPK (3) and mitochondrial AMPK (4) are activated independently of AXIN, leading to phosphorylation of ACC2 and MFF and accelerating catabolic activities, along with all the other substrates that can be phsophorylated at lower AMP levels. Of note, TSC2 and Raptor can be phosphorylated by all the four modes to respectively inhibit mTORC1 activity. Experiments in this figure were performed three times. See also Supplementary information, Fig. S4

Biotechnology. The HRP-conjugated goat anti-mouse lgG (cat. 115-035-003, 1:5000 for IB) and HRP-conjugated goat anti-rabbit IgG (cat. 111-035-003, 1:5000 for IB) antibodies were purchased from Jackson ImmunoResearch.

Bacterial and virus strains

Stbl3 (cat. C737303, for transformation of pBOBI- and pLL3.7based constructs), BL21 (DE3, cat. C600003, for transformation of pET-based constructs) and DH5a (cat. 18258012, for transformation of other constructs) competent cells were purchased from Thermo Fisher Scientific.

Chemicals and recombinant proteins

TOFA (cat. sc-200653A) was purchased from Santa Cruz Biotechnology. AICAR (cat. A9978), AMP (cat. 01930) and LKB1 complex (cat. SRP0246) were purchased from Sigma. A-769662 (cat. S2697) was purchased from Selleck. Lysosome Isolation Kit (cat. LYSISO1) was purchased from Sigma.

Cell lines

HEK293T cells (cat. CRL-3216) were obtained from ATCC, and HEK293 cells (cat. CRL-1573) were obtained from Invitrogen. LAMTOR $1^{\mathrm{F} / \mathrm{F}}$ or $A X I N^{\mathrm{F} / \mathrm{F}}$ MEFs were established by introducing SV40 $T$ antigen into primary cultured embryonic cells from a litter of corresponding mice.

\section{Oligonucleotides}

The siRNAs against human AXIN1 and LAMTOR1 were constructed and validated as described previously. ${ }^{18,19}$ The sequence for each sgRNA is as follows (forward strand only):

5'- TAGGATCGGAAACTTACTAGTGG $-3^{\prime}$ and $5^{\prime}$ - GGCTCAGGTTAA CTAACGT

TAGG $-3^{\prime}$ for human PRKAB1,

5'- TITAGGCAGTGCTTGAGCATAGG $-3^{\prime}$ and $5^{\prime}$ - CGGCCACTTAG

TATATCTGT

GGG $-3^{\prime}$ for human PRKAB2,

5'- CTGATTIATAGTAGCGGTGCAGG-3' for human PRKAG1,

$5^{\prime}$ - GATGCAGTCACTCCACGCTCTGG $-3^{\prime}$ and $5^{\prime}$ - CGGTGGCAC CGAAGCTGC

CAGG-3' for human PRKAG2,

5'-AGCCCGTGCGCTCAATCTTCTGG-3' and $5^{\prime}$-TGGGGCCTGTाTG GTTAATA

GGG-3' for human PRKAG3,

and 5'-CACCGAGTCAGCTCTGGATCGGAGA-3' for mouse Ak1.

Animals

$A X I N^{\mathrm{F} / \mathrm{F}}$, LAMTOR $1^{\mathrm{F} / \mathrm{F}}$ mice were generated and maintained as described. ${ }^{18}$ The ACC1-floxed mouse (Stock No. 030954, The
Jackson Laboratory) was a generous gift from Prof. Jay Horton (UT Southwestern Medical Center), and the $A C C 2^{-1-}$ mouse from Prof. Tian Xu (Institute of Developmental Biology and Molecular Medicine, Fudan University). Wildtype C57BL/6J mice were purchased from Beijing Vital River Laboratory Animal Technology Co., Ltd., and were housed at Animal Core Facility. Male littermate mice of 6-week old were used in this study. Mice were housed with free access to water and fed a standard diet $(65 \%$ carbohydrate, $11 \%$ fat, $24 \%$ protein). Protocols for all animal experiments were approved by the Institutional Animal Care and the Animal Committee of Xiamen University.

\section{Mouse studies}

For starvation, the diet was withdrawn from the cage at 5 p.m. and mice were sacrificed at 9 a.m. the next day by cervical dislocation. For hepatic ischemia, surgery was performed as described previously ${ }^{72}$ with some modifications. Mice were anesthetized with pentobarbital sodium solution $(100 \mathrm{mg} / \mathrm{kg})$ by intraperitoneal injection and were placed on a heating plate set at $37^{\circ} \mathrm{C}$. The mouse abdomen was gently opened (from the mid-abdomen to the xiphoid) to expose the liver, and its intestine was covered by moistened gauze to prevent from drying. The portal vein, hepatic artery, and bile duct were then cross-clamped by an atraumatic clip at a place just above the branching to the right lateral lobe to subject the median and the left lateral lobes to ischemia. Mice that underwent the same surgery but did not undergo cross-clamping on the blood vessel and bile duct were considered as shams. Unless stated otherwise, liver tissues were dissected and instantly frozen in liquid nitrogen for immunoblotting, and were freezeclamped for extraction and fractionation of metabolites.

Plasmids

Point mutations on AMPK- $\gamma 1$ (D317A), AMPK- $\gamma 2$ (R531G), and AMPK- $\beta 1$ (G2A) were made using a PCR-based site-directed mutagenesis method using PrimeSTAR HS polymerase (Takara). Expression plasmids for various proteins were constructed in the pcDNA3.3 vector for transient transfection (ectopic expression), in pBOBI for lentivirus packaging (stable expression). PCR products were verified by sequencing (Invitrogen, China). The lentivirusbased vector pLL3.7 was used for expression of siRNA in HEK293T and HEK293 cells.

CRISPR/Cas9 knockout cell lines

Genes were deleted in HEK293T cells (human PRKAB1, PRKAB2, PRKAG1, PRKAG2, and PRKAG3) or MEFs (mouse Ak1) using the CRISPR-Cas9 system. Targeting nucleotides were designed using http://crispr.mit.edu. Oligonucleotides of human PRKAG1 were inserted into pX260 vector, human PRKAB1, PRKAB2, PRKAG2, and 
470

PRKAG3 into pX330 vector, and mouse AK1 into the lentiCRISPRv2 vector. The individual constructs were then subjected to transfection of HEK293T cells (pX260 and pX330) followed by single-cell sorting into 96-well dishes, or lentivirus packaging (lentiCRISPRv2) using HEK293T cells. In both conditions, cells were transfected with $3 \mu \mathrm{g}$ of DNA with Lipofectamine 2000 (Invitrogen, Cat. 11668027) per well of a 6-well plate. Clones were expanded and evaluated for knockout status by sequencing.

Cell culture, transient transfection and lentivirus Infection HEK293T, HEK293 and MEFs were maintained in Dulbecco's modified Eagle's medium (DMEM, Gibco, cat. 11965) supplemented with $10 \%$ fetal bovine serum (FBS), $100 \mathrm{IU}$ penicillin, $100 \mathrm{mg} /$ $\mathrm{ml}$ streptomycin at $37{ }^{\circ} \mathrm{C}$ in a humidified incubator containing $5 \%$ $\mathrm{CO}_{2}$. Polyethylenimine (Polysciences, Inc., Cat. \#23966) at a final concentration of $10 \mu \mathrm{M}$ was used to transfect HEK293T cells. Total DNA for each plate was adjusted to the same amount by using relevant empty vector. Transfected cells were harvested at $24 \mathrm{~h}$ after transfection. Lentiviruses for infection of the MEFs were packaged in HEK293T cells using Lipofectamine 2000 transfection. At $30 \mathrm{~h}$ post transfection, medium was collected and added to the cells. The cells were incubated for another $24 \mathrm{~h}$. LAMTOR ${ }^{\mathrm{F} / \mathrm{F}}$ or $A X I N^{\mathrm{F} / F}$ MEFs were established by introducing SV40 T antigen into primary cultured embryonic cells from a mouse litter. LAMTOR $1^{-1-}$ or $A X I N^{-1-}$ MEFs were generated by infecting $L A M T O R 1^{\mathrm{F} / \mathrm{F}}$ or $A X I N^{\mathrm{F} / F}$ MEFs with adenovirus expressing Cre recombinase for 12 $h$. The infected cells were then incubated in fresh DMEM for another $8-10 \mathrm{~h}$ before further treatments. Cells were verified to be free of mycoplasma contamination and authenticated by STR sequencing.

For glucose starvation, cells were rinsed twice with PBS, and then incubated in glucose-free DMEM (Gibco, cat. 11966) supplemented with $10 \%$ FBS and $1 \mathrm{mM}$ sodium pyruvate (Gibco, cat. 11360) for desired periods of time at $37^{\circ} \mathrm{C}$. For starvation for glucose plus glutamine, cells were incubated in DMEM with $5 \mathrm{mM}$ glucose overnight, and then incubated for desired periods of time in fresh DMEM lacking glucose, glutamine, phenol red or pyruvate (Gibco cat. A14430-01), in which $1 \mathrm{mM}$ pyruvate was added, with or without Glutamax ${ }^{\mathrm{TM}}$ (Gibco cat. 35050-038) and D-glucose (Gibco cat. A2494001), all without FBS.

\section{Subcellular fractionation}

All buffers used for subcellular fractionation contained a protease inhibitor cocktail (Sigma, cat. P8340).

Lysosomes were purified by Lysosome Isolation Kit according to the manufacturer's instructions with minor modifications. Briefly, cells from sixty $10-\mathrm{cm}$ dishes (60-80\% confluence) were collected by direct scraping at room temperature, followed by centrifugation for $5 \mathrm{~min}$ at $500 \mathrm{~g}$ at $37^{\circ} \mathrm{C}$. Cells were resuspend in $7 \mathrm{ml}$ of $1 \times$ Extraction Buffer at room temperature. For isolating lysosomes from mouse livers, approximately $500 \mathrm{mg}$ of frozen liver tissue was directly homogenized in $7 \mathrm{ml}$ of ice-cold $1 \times$ Extraction Buffer. The cell/liver homogenates were then homogenized in a 7-ml Dounce homogenizer (Sigma, cat. P0610) for 120 strokes on ice followed by centrifuging for $10 \mathrm{~min}$ at $1000 \mathrm{~g}, 4^{\circ} \mathrm{C}$ to yield post-nuclear supernatants (PNS). The PNS were then centrifuged for $20 \mathrm{~min}$ at $20,000 \mathrm{~g}$ and the pellets were suspended in 1× Extraction Buffer by gentle pipetting to generate Crude Lysosomal Fractions (CLF). The volume of CLF was adjusted to $2.4 \mathrm{ml}$ and then equally divided into six $1.5 \mathrm{ml}$ Eppendorf tubes ( $400 \mu \mathrm{l}$ per tube). $253 \mu \mathrm{l}$ of OptiPrep and $137 \mu$ l of $1 \times$ OptiPrep Dilution Buffer were added to each CLF and mixed by gentle pipetting. The mixtures were defined as the Diluted OptiPrep Fraction (DOF). Each DOF $(0.8 \mathrm{ml})$ was loaded onto an $11 \times 60 \mathrm{~mm}$ centrifuge tube at the top of $27 \%$ $(0.4 \mathrm{ml})$ and $22.5 \%(0.5 \mathrm{ml})$ OptiPrep solution cushions, and then overlaid with $16 \%(1 \mathrm{ml}), 12 \%(0.9 \mathrm{ml})$ and $8 \%(0.3 \mathrm{ml})$ OptiPrep solutions. The tubes were then centrifuged on a SW60 Ti rotor (Beckman) at $150,000 \mathrm{~g}$ for $4 \mathrm{~h}$ at $4{ }^{\circ} \mathrm{C}$ and the fractions at the top of $12 \%$ OptiPrep solution were collected as the crude lysosome fractions. The fractions were diluted with two volumes of PBS, followed by centrifugation at $20,000 \mathrm{~g}$ for $20 \mathrm{~min}$. The sediment was the lysosome fraction.

Mitochondria were purified as described previously ${ }^{73}$ with minor modifications. Briefly, forty $10-\mathrm{cm}$ dishes of regularly cultured, severely starved or AICAR-treated cells $(60-80 \%$ confluence), or sixty $10-\mathrm{cm}$ dishes of glucose-starved cells were collected by direct scraping at room temperature, followed by centrifugation for $5 \mathrm{~min}$ at $500 \mathrm{~g}$ at $37^{\circ} \mathrm{C}$. Cells were then resuspended in $20 \mathrm{ml}$ of ice-cold $\mathrm{IB}_{\text {cells }}-1$ buffer ( $225 \mathrm{mM}$ mannitol, $75 \mathrm{mM}$ sucrose, $0.1 \mathrm{mM}$ EGTA and $30 \mathrm{mM}$ Tris- $\mathrm{HCl}, \mathrm{pH}$ 7.4). To isolate mitochondria from mouse livers, approximately $500 \mathrm{mg}$ of frozen liver was directly homogenized in $10 \mathrm{ml}$ of $\mathrm{IB}_{\text {liver }}-1$ buffer (225 mM mannitol, $75 \mathrm{mM}$ sucrose, 0.5\% BSA, 0.5 mM EGTA and $30 \mathrm{mM}$ Tris- $\mathrm{HCl}, \mathrm{pH}$ 7.4). Cells or liver homogenates were then homogenized in a 40-ml Dounce homogenizer (Sigma, cat. D9188) for 100 strokes, followed by two rounds of centrifugation for $5 \mathrm{~min}$ at $600 \mathrm{~g}$ (for cells) or $740 \mathrm{~g}$ (for liver tissues) at $4{ }^{\circ} \mathrm{C}$. The supernatants were then collected and centrifuged for $10 \mathrm{~min}$ at $7000 \mathrm{~g}$ (for cells) or $9000 \mathrm{~g}$ (for liver tissues) at $4{ }^{\circ} \mathrm{C}$. The pellets were then washed with $20 \mathrm{ml}$ of ice-cold $\mathrm{IB}_{\text {cells }}-2$ buffer (for cells, $225 \mathrm{mM}$ mannitol, $75 \mathrm{mM}$ sucrose and $30 \mathrm{mM}$ Tris- $\mathrm{HCl} \mathrm{pH} 7.4$ ) or ice-cold $\mathrm{IB}_{\text {liver }}-2$ buffer (for liver tissues, $\mathrm{IB}_{\text {cells }}-2$ buffer supplemented with $0.5 \% \mathrm{BSA}$ ) twice. The suspensions were first centrifuged at $7000 \mathrm{~g}$ (for cells) or $10,000 \mathrm{~g}$ (for liver tissues) and were centrifuged again at $10,000 \mathrm{~g}$, both for $10 \mathrm{~min}$ at $4{ }^{\circ} \mathrm{C}$. Note that for liver tissues, the pellets subjected to the second time of centrifugation were resuspended in $20 \mathrm{ml}$ of ice-cold $\mathrm{IB}_{\text {cells }}-2$. The pellets were then resuspended in $2 \mathrm{ml}$ of ice-cold MRB buffer (250 mM mannitol, $5 \mathrm{mM}$ HEPES pH 7.4 and $0.5 \mathrm{mM}$ EGTA) and were loaded on top of $10 \mathrm{ml}$ of Percoll medium ( $225 \mathrm{mM}$ mannitol, $25 \mathrm{mM}$ HEPES pH 7.4, $1 \mathrm{mM}$ EGTA and 30\% Percoll (v/v)) in $14 \times$ 89-mm centrifuge tubes (Beckman, ref. 344059). The tubes were then centrifuged on a SW41 rotor (Beckman) at 95,000 $\mathrm{g}$ for $0.5 \mathrm{~h}$ at $4{ }^{\circ} \mathrm{C}$, and the dense band located approximately at the bottom of each tube was collected. The collected fractions were diluted with 10 volumes of MRB buffer, followed by centrifugation at $6300 \mathrm{~g}$ for $10 \mathrm{~min}$ at $4^{\circ} \mathrm{C}$ and washed with $2 \mathrm{ml}$ of MRB buffer, followed by centrifugation at $6300 \mathrm{~g}$ for $10 \mathrm{~min}$ at $4^{\circ} \mathrm{C}$. The pellets contained purified mitochondria.

Cytosol was purified as described. ${ }^{74}$ Briefly, some $0.15 \mathrm{~g}$ of each freshly excised liver tissue or ten $10-\mathrm{cm}$ dishes of cells were homogenized in $800 \mu \mathrm{l}$ of the homogenization buffer (HB) containing $250 \mathrm{mM}$ sucrose, $3 \mathrm{mM}$ imidazole, $\mathrm{pH}$ 7.4. Homogenates were then passed through a $22-\mathrm{G}$ needle attached to a $1-\mathrm{ml}$ syringe for six times, and were then centrifuged at $2000 \mathrm{~g}$ for 10 min to yield PNS. PNS samples were then loaded on the top of $11 \times 60-\mathrm{mm}$ centrifuge tubes that had been loaded sequentially with $1 \mathrm{ml}$ of $40.6 \%$ sucrose (dissolved in HB), $1 \mathrm{ml}$ of $35 \%$ sucrose (dissolved in $\mathrm{HB}$ ), and $1 \mathrm{ml}$ of $25 \%$ sucrose (dissolved in $\mathrm{HB}$ ). Tubes were then centrifuged on an SW60 Ti rotor (Beckman) at 35,000 rpm for $1 \mathrm{~h}$ at $4{ }^{\circ} \mathrm{C}$, and the top fractions (about $200 \mu \mathrm{l}$ ) were collected as cytosolic fraction.

Fractionation of nuclei was performed as described previously. ${ }^{19}$ Briefly, cells were homogenized in Buffer A $(10 \mathrm{mM}$ HEPES, pH 7.9, $10 \mathrm{mM} \mathrm{KCl}, 0.1 \mathrm{mM}$ EDTA, $0.1 \mathrm{mM}$ EGTA, and 0.15\% NP-40) and placed in ice for $15 \mathrm{~min}$. The homogenates were centrifuged at $12,000 \mathrm{~g}$ for $1 \mathrm{~min}$ at $4{ }^{\circ} \mathrm{C}$. The pellets were washed three times with Buffer $A$ and then resuspended in Buffer $B(20$ mM HEPES, pH 7.9, $0.4 \mathrm{mM} \mathrm{NaCl} 1 \mathrm{mM}$ EDTA, $1 \mathrm{mM}$ EGTA, 0.5\% NP-40) and sonicated at $4{ }^{\circ} \mathrm{C}$. Cellular debris was removed by centrifugation at $12,000 \mathrm{~g}$ for $30 \mathrm{~min}$ at $4{ }^{\circ} \mathrm{C}$, and the supernatant was nuclear fraction.

Protein production

AXIN1, and AMPK complexes were expressed and purified in E. coli as described previously. ${ }^{19}$ Briefly, full length AXIN1 was cloned 
into $\mathrm{pET} 32 \mathrm{a}$ vector and transformed into the E. coli strain BL21 (DE3). The transformed cells were induced with $0.1 \mathrm{mM}$ IPTG when the culture attained an optical density of 0.4 at $600 \mathrm{~nm}$ (for AMPK, to 1.0 at $600 \mathrm{~nm}$ ). After growing for $4 \mathrm{~h}$ at $16^{\circ} \mathrm{C}$ (for AMPK, growing for $16 \mathrm{~h}$ ), the cells were collected, homogenized in buffer (50 mM sodium phosphate, pH 7.4, $150 \mathrm{mM} \mathrm{NaCl}, 1 \%$ Triton X-100, $5 \%$ glycerol) and sonicated. Cell debris was removed by centrifugation. The supernatant was collected and subjected to ultracentrifugation at $150,000 \mathrm{~g}$ for $30 \mathrm{~min}$, followed by purification of expressed protein with Nickel affinity gel and washed by $50 \mathrm{mM}$ sodium phosphate, $\mathrm{pH} 7.4,150 \mathrm{mM} \mathrm{NaCl}$. The protein was eluted from the affinity resin by $50 \mathrm{mM}$ sodium phosphate, $\mathrm{pH} 7.4$, $150 \mathrm{mM} \mathrm{NaCl}, 250 \mathrm{mM}$ imidazole, and concentrated to about $3 \mathrm{mg} / \mathrm{ml}$ before further purification by gel filtration (Superdex-200, GE Healthcare). The buffer for gel filtration contained $50 \mathrm{mM}$ Tris$\mathrm{HCl}, \mathrm{pH} 7.4,150 \mathrm{mM} \mathrm{NaCl}, 5 \%$ glycerol and $1 \mathrm{mM}$ DTT.

In vitro AMPK phosphorylation assay

Bacterially expressed and purified AMPK (800 ng) was incubated with active LKB1/MO25/STRAD complex (200 ng) at $32{ }^{\circ} \mathrm{C}$ for 15 min in a kinase buffer containing $5 \mathrm{mM}$ ATP with or without 60 $\mu \mathrm{M}$ AMP and $1 \mu \mathrm{g}$ of AXIN1. Phosphorylation of AMPK was determined by immunoblotting.

Immunoprecipitation (IP) and immunoblotting

IP of endogenous AMPKa was performed as described previously ${ }^{19}$ and was also used for IP ACC1 and ACC2, although with some modifications. Briefly, cells from a single $15-\mathrm{cm}$ dish (grown to $80 \%$ confluence) or $50 \mathrm{mg}$ liver (for each lane) were collected and lysed with $500 \mu \mathrm{l}$ of ice-cold lysis buffer $(20 \mathrm{mM}$ Tris- $\mathrm{HCl}, \mathrm{pH}$ 7.5, $1 \mathrm{mM}$ EDTA, $1 \mathrm{mM}$ EGTA, $1 \%$ Triton X-100, $1 \mathrm{mM} \beta-$ glycerolphosphate, with protease inhibitor cocktail. Note that $150 \mathrm{mM} \mathrm{NaCl}$ was used for IP of ACC1 and ACC2, and $50 \mathrm{mM} \mathrm{NaCl}$ was used for IP of AMPKa. Lysates were incubated with respective antibodies for $4 \mathrm{~h}$ (ACC1 and ACC2) or overnight (AMPKa). Protein aggregates were pre-cleared by centrifugation at $20,000 \mathrm{~g}$ for 10 min, and protein A/G beads (1:100) were then added into the lysates and mixed for another 1 (for ACC1 and ACC2) to $3 \mathrm{~h}$ (for AMPKa). The beads were washed with $100 x$ volumes of lysis buffer for 3 times at $4{ }^{\circ} \mathrm{C}$ and then mixed with an equal volume of $2 \times$ SDS sample buffer for immunoblotting.

To analyze the levels of p-AMPKa and p-ACC in MEFs, cells grown to $70-80 \%$ confluence in a well of a 6-well dish were lysed with $250 \mu \mathrm{l}$ of ice-cold lysis buffer. The lysates were then centrifuged at $20,000 \mathrm{~g}$ for $10 \mathrm{~min}$ at $4{ }^{\circ} \mathrm{C}$ and an equal amount of $2 \times$ SDS sample buffer was into each supernatant. The levels of p-AMPKa and p-ACC were then analyzed by immunoblotting. To analyze the levels of p-AMPKa and p-ACC in liver, the freshly excised liver tissues were added with ice cold lysis buffer $(10 \mu \mathrm{l} /$ mg liver weight), followed by homogenization and centrifugation as described above. The lysates were then mixed with $2 \times$ SDS sample buffer and then subjected to immunoblotting. Levels of total proteins and the levels of phosphorylation of proteins were analyzed on separate gels and representative images were shown. The band intensities on developed films were quantified using Image J software (National Institutes of Health Freeware).

\section{Fluorescence microscopy}

HEK293T infected with different fusion constructs of $\beta 1$ were grown on glass coverslips in 6-well dishes and were cultured to $60-80 \%$ confluence. Cells were fixed with $1 \mathrm{ml}$ of $4 \%$ formaldehyde (diluted in PBS) at room temperature for $20 \mathrm{~min}$. They were rinsed twice with $1 \mathrm{ml}$ PBS (room temperature) and then permeabilized with $1 \mathrm{ml}$ of $0.1 \%$ Triton X-100 (diluted in PBS) for $5 \mathrm{~min}$ at $4{ }^{\circ} \mathrm{C}$. Cells were rinsed twice with $1 \mathrm{ml} \mathrm{PBS}$, and were incubated with primary antibodies overnight at $4{ }^{\circ} \mathrm{C}$. The cells were then rinsed three times with $1 \mathrm{ml} \mathrm{PBS}$, and then incubated with Alexa-Fluor 594-conjugated anti mouse secondary antibody
(Molecular Probes, cat. A11032; diluted 1:100 in PBS) and AlexaFluor 488-conjugated anti-rabbit secondary antibody (Molecular Probes, R37117; diluted 1:100 in PBS) for $8 \mathrm{~h}$ at room temperature in the dark. They were washed four times with $1 \mathrm{ml} \mathrm{PBS}$, and then mounted on glass slides using ProLong Diamond Antifade Mountant (Molecular Probes, cat. P36970). Cells were imaged under a Zeiss LSM 780. Samples were excited with an Ar gas laser (Zeiss, laser module LGK 7812) using a 488-nm laser line for AlexaFluor 488 dye (green channel), and with a HeNe gas laser (Zeiss, LGK 7512 PF) using a 594-nm laser line for Alexa-Fluor 594 dye (red channel). Confocal microscope images were taken with a $63 \times$ oil objective and representative images were shown. The parameters, including 'PMT voltage', 'Offset', 'Pinhole' and 'Gain', were kept unchanged between each image taken.

\section{CE-MS-based analysis of AMP, ADP, and ATP}

Sample preparation for CE (capillary electrophoresis)-MS was carried out as described previously, ${ }^{75,76}$ with some modifications. In general, each measurement required cells to be collected from a $10-\mathrm{cm}$ dish (60-70\% confluence) or $100 \mathrm{mg}$ of liver tissue. For analysis of metabolites, cells were rinsed with $20 \mathrm{ml}$ of $5 \%$ mannitol solution (dissolved in water) and instantly frozen in liquid nitrogen. Cells were then lysed with $1 \mathrm{ml}$ of methanol containing internal standards 1 [IS1 (Human Metabolome Technologies, H3304-1002, 1:200), used to standardize the metabolite intensity and to adjust the migration time], and were scraped from the dish. For analysis of metabolites in liver, the fleshly excised tissue was freeze-clamped first, then washed in pre-cooled $5 \%$ mannitol solution and grinded in $1 \mathrm{ml}$ of methanol with $50 \mu \mathrm{M}$ IS1. The lysate was then mixed with $1 \mathrm{ml}$ of chloroform and $400 \mu \mathrm{l}$ of water by $20 \mathrm{~s}$ of vortexing. After centrifugation at $15,000 \mathrm{~g}$ for $15 \mathrm{~min}$ at $4{ }^{\circ} \mathrm{C}, 450 \mu \mathrm{l}$ of aqueous phase was collected and was then filtrated through a $5 \mathrm{kDa}$ cutoff filter (Millipore, cat. UFC3LCCNB-HMT) by centrifuging at $10,000 \mathrm{~g}$ for $3 \mathrm{~h}$ at $4{ }^{\circ} \mathrm{C}$. Simultaneously, the quality control (QC) sample was prepared by combining $100 \mu \mathrm{l}$ of the aqueous phase from each samples and then filtered. The filtered aqueous phase was then freeze-dried in a vacuum concentrator and then dissolved in water containing internal standards 3 [IS3 (Human Metabolome Technologies, H3304-1104, 1:200), to adjust the migration time]. $20 \mu \mathrm{l}$ of redissolved solution was then loaded into an injection vial with a conical insert for CE-TOF MS (Agilent Technologies 7100, equipped with 6224 mass spectrometer) analysis. For quantification of AMP and ATP, $\left[U-{ }^{13} \mathrm{C},{ }^{15} \mathrm{~N}\right] \mathrm{AMP}$ and $\left[\mathrm{U}-{ }^{13} \mathrm{C},{ }^{15} \mathrm{~N}\right] \mathrm{ATP}$ were used to generate standard curves by plotting the ratios of detected labelled AMP or ATP (areas) to the products of IS1 and IS3, against the added concentrations of labelled AMP or ATP. The standard curve of $\left[\mathrm{U}^{13} \mathrm{C}^{15} \mathrm{~N}\right] \mathrm{AMP}$ was also used for quantification of ZMP, because of the structural similarity between them. The amount of AMP, ZMP, and ATP were then estimated according to equations generated from standard curves. The average cell volume, 2263 $\mu \mathrm{m}^{3}$, was determined by Imaris 7.4.0 software (Bitplane) from the axial image stacks of CDFA-SE labelled MEFs taken under Zeiss LSM780.

HPLC-MS-based analysis of short-chain acyl-CoA

Procedure of preparing samples for HPLC-MS-based acyl-CoA analysis was identical to that described in the CE-MS section, except that cells or liver tissues were rinsed in PBS, and that ${ }^{13} \mathrm{C} 2: 0-\mathrm{CoA}(150 \mu \mathrm{g} / \mathrm{l})$ and ${ }^{13} \mathrm{C} 3: 0-\mathrm{CoA}(200 \mu \mathrm{g} / \mathrm{l})$ prepared in methanol were added as internal standards. Some $800 \mu \mathrm{l}$ of the aqueous phase of each sample was collected after centrifugation at $15,000 \mathrm{~g}$ for $15 \mathrm{~min}$ at $4{ }^{\circ} \mathrm{C}$ and was lyophilized in a vacuum concentrator. Samples were then re-dissolved in $20 \mu$ of ice-cold $20 \%$ methanol. Measurement of acetyl- and malonyl-CoAs was based on a previous study ${ }^{77}$ using a QTRAP (SCIEX, QTRAP 6500 plus) mass spectrometer interfaced with a UPLC system (Waters, ACQUITY UPLC system). Some $5 \mu$ l of each sample were injected 
onto an Acquity HSS T3 column $(2.1 \times 50 \mathrm{~mm}, 1.7 \mu \mathrm{m}$, Waters $)$ attached to an Acquity BEH C18 pre-column $(2.1 \times 5 \mathrm{~mm}, 1,7 \mu \mathrm{m}$, Waters). Mobile phases A and B were water and acetonitrile, respectively (both contains $10 \mathrm{mM}$ ammonium acetate and $0.05 \%$ ammonium hydroxide). The column temperature was maintained at $30^{\circ} \mathrm{C}$ and autosampler temperature at $8{ }^{\circ} \mathrm{C}$. The gradients were as follows: $t=0 \mathrm{~min}, 3 \% \mathrm{~B} ; t=1 \mathrm{~min}, 3 \% \mathrm{~B} ; t=13 \mathrm{~min}, 15 \% \mathrm{~B} ; t=$ $14.1 \mathrm{~min}, 100 \% \mathrm{~B} ; t=14.7 \mathrm{~min}, 100 \% \mathrm{~B} ; t=14.71 \mathrm{~min}, 3 \% \mathrm{~B} ; t=17$ $\min , 3 \% \mathrm{~B}$. Mass spectrometry was in positive mode with multiple reactions monitoring mode (MRM), declustering potentials (DP) and collision energies (CE) set at 200 and $38 \mathrm{~V}$, respectively (optimized using ${ }^{13} \mathrm{C} 2: 0-$ and ${ }^{13} \mathrm{C} 3: 0-\mathrm{CoA}$ ). The following transitions and retention time were used for monitoring each compound: $812.0,305.3$, and $2.2 \mathrm{~min}$ for ${ }^{13} \mathrm{C} 2: 0-\mathrm{CoA}, 810.1$, 303.1, and $2.2 \mathrm{~min}$ for acetyl-CoA, 856.9, 350.2, and $0.8 \mathrm{~min}$ for ${ }^{13} \mathrm{C} 3: 0-\mathrm{CoA}$, and $854.1,347.1$, and $0.8 \mathrm{~min}$ for malonyl-CoA. It should be noted that acyl-CoAs were extremely easy to degrade even frozen at $-80^{\circ} \mathrm{C}$, so no pause was taken during the whole analytic process and no more than 6 samples were re-dissolved and kept in autosampler at one time.

\section{Statistical analysis}

One-way or two-way ANOVA with post hoc analysis was used to compare values among different experimental groups. For experiments with only two groups, a two-tailed Student's $t$ test was used as specified in the figure legends. For ANOVA, the homogeneity of variance was firstly tested by Levene's test. If the results were similar, the Tukey's test was preceded, and if not, the Games-Howell's test was processed. Similar procedures were followed when the Student's $t$ test was performed. No samples or animals were excluded from the analysis. Tests were performed with SPSS Statistics 17.0 program, and $p<0.05$ was considered statistically significant. Statistical significance is shown as ${ }^{*} p<0.05$, ${ }^{* *} p<0.01,{ }^{* * *} p<0.001$; N.S., not significant.

\section{ACKNOWLEDGEMENTS}

ACC1-floxed mouse (Stock No. 030954, The Jackson Laboratory) was a generous gift from Prof. Jay Horton (UT Southwestern Medical Center), and the $A C C 2^{-1-}$ mouse from Prof. Tian Xu (Institute of Developmental Biology and Molecular Medicine, Fudan University). Heterotrimeric AMPK cloned into pET21b was a generous gift from Prof. Dietbert Neumann (Swiss Federal Institute of Technology). We also thank Jiayuan Zhang from Xiamen University for the artwork of Fig. 4a. This work was supported by grants from the National Key R\&D Program of China (2016YFA0502001) and the National Natural Science Foundation of China (\#31730058, \#31430094, \#31601152 and \#31690101) through S-C.L., by the Open Research Fund of State Key Laboratory of Cellular Stress Biology, Xiamen University (SKLCSB2017KF002) through H-L.P., and by Wellcome Trust (204766/Z/16/Z) through D.G.H.

\section{AUTHOR CONTRIBUTIONS}

Y.Z., C-S.Z., M.L., and S-C.L. conceived the study and designed the experiments. Y.Z., C-S.Z., and M.L. performed the subcellular fractionation, immunoprecipitation, in vitro reconstitution and the associated western blot analyses with assistance from S.A.H, T. M., J.-W.F., X.T., Q.Q., and Y.-Q.W. Y.Z., W.W., Z.W., and C.Z. performed the CE-MS- and HPLC-MS-based analysis of adenylates and CoAs under the guidance of H.-L.P. M.L. performed the confocal imaging acquisition. Z.Y., S-Y.L., H.-L.P., and D.G.H. helped with discussion and interpretation of results. D.G.H. and S-C.L. wrote the manuscript.

\section{ADDITIONAL INFORMATION}

Supplementary information accompanies this paper at https://doi.org/10.1038/ s41422-019-0163-6.

Competing interests: The authors declare no competing interests.

\section{REFERENCES}

1. Carling, D., Thornton, C., Woods, A. \& Sanders, M. J. AMP-activated protein kinase: new regulation, new roles? Biochem. J. 445, 11-27 (2012).
2. Steinberg, G. R. \& Kemp, B. E. AMPK in Health and Disease. Physiol. Rev. 89, 1025-1078 (2009).

3. Herzig, S. \& Shaw, R. J. AMPK: guardian of metabolism and mitochondrial homeostasis. Nature reviews. Mol. Cell Biol. https://doi.org/10.1038/nrm.2017.95 (2017).

4. Lin, S. C. \& Hardie, D. G. AMPK: sensing glucose as well as cellular energy status. Cell Metab. 27, 299-313 (2017).

5. Viollet, B. et al. AMPK inhibition in health and disease. Crit. Rev. Biochem. Mol. Biol. 45, 276-295 (2010).

6. Scott, J. W. et al. CBS domains form energy-sensing modules whose binding of adenosine ligands is disrupted by disease mutations. J. Clin. Invest. 113, 274-284 (2004).

7. Xiao, B. et al. Structural basis for AMP binding to mammalian AMP-activated protein kinase. Nature 449, 496-500 (2007).

8. Gu, X. et al. Deconvoluting AMP-dependent kinase (AMPK) adenine nucleotide binding and sensing. J. Biol. Chem. 292, 12653-12666 (2017).

9. Ross, F. A., Jensen, T. E. \& Hardie, D. G. Differential regulation by AMP and ADP of AMPK complexes containing different gamma subunit isoforms. Biochem. J. 473, 189-199 (2016).

10. Hawley, S. A. et al. Calmodulin-dependent protein kinase kinase-beta is an alternative upstream kinase for AMP-activated protein kinase. Cell Metab. 2, 9-19 (2005).

11. Woods, A. et al. $\mathrm{Ca}^{+} /$calmodulin-dependent protein kinase kinase-beta acts upstream of AMP-activated protein kinase in mammalian cells. Cell Metab. 2, 21-33 (2005).

12. Hurley, R. L. et al. The $\mathrm{Ca}^{2+} /$ calmoldulin-dependent protein kinase kinases are AMP-activated protein kinase kinases. J. Biol. Chem. 280, 29060-29066 (2005).

13. Salt, I. P., Johnson, G., Ashcroft, S. J. H. \& Hardie, D. G. AMP-activated protein kinase is activated by low glucose in cell lines derived from pancreatic b cells, and may regulate insulin release. Biochem. J. 335, 533-539 (1998).

14. Zhang, C. S. et al. Fructose-1,6-bisphosphate and aldolase mediate glucose sensing by AMPK. Nature 548, 112-116 (2017).

15. Gonzalez, P. S. et al. Mannose impairs tumour growth and enhances chemotherapy. Nature 563, 719-723 (2018).

16. Nada, S. et al. The novel lipid raft adaptor p18 controls endosome dynamics by anchoring the MEK-ERK pathway to late endosomes. EMBO J. 28, 477-489 (2009).

17. Bar-Peled, L., Schweitzer, L. D., Zoncu, R. \& Sabatini, D. M. Ragulator is a GEF for the rag GTPases that signal amino acid levels to mTORC1. Cell 150, 1196-1208 (2012).

18. Zhang, C. S. et al. The lysosomal v-ATPase-ragulator complex is a common activator for AMPK and mTORC1, acting as a switch between catabolism and anabolism. Cell Metab. 20, 526-540 (2014).

19. Zhang, Y. L. et al. AMP as a low-energy charge signal autonomously initiates assembly of AXIN-AMPK-LKB1 complex for AMPK activation. Cell Metab. 18, 546-555 (2013).

20. Miyamoto, T. et al. Compartmentalized AMPK signaling illuminated by genetically encoded molecular sensors and actuators. Cell Rep. 11, 657-670 (2015).

21. Salt, I. P. et al. AMP-activated protein kinase-greater AMP dependence, and preferential nuclear localization, of complexes containing the a2 isoform. Biochem. J. 334, 177-187 (1998).

22. Kazgan, N., Williams, T., Forsberg, L. J. \& Brenman, J. E. Identification of a nuclear export signal in the catalytic subunit of AMP-activated protein kinase. Mol. Biol. Cell 21, 3433-3442 (2010).

23. Vincent, O., Townley, R., Kuchin, S. \& Carlson, M. Subcellular localization of the Snf1 kinase is regulated by specific beta subunits and a novel glucose signaling mechanism. Genes Dev. 15, 1104-1114 (2001).

24. Liang, J. et al. Myristoylation confers noncanonical AMPK functions in autophagy selectivity and mitochondrial surveillance. Nat. Commun. 6, 7926 (2015).

25. $\mathrm{Yi}, \mathrm{C}$. et al. Formation of a Snf1-Mec1-Atg1 module on mitochondria governs energy deprivation-induced autophagy by regulating mitochondrial respiration. Dev. Cell 41, 59-71 e54 (2017).

26. Tsou, P., Zheng, B., Hsu, C. H., Sasaki, A. T. \& Cantley, L. C. A fluorescent reporter of AMPK activity and cellular energy stress. Cell Metab. 13, 476-486 (2011).

27. Warden, S. M. et al. Post-translational modifications of the beta-1 subunit of AMPactivated protein kinase affect enzyme activity and cellular localization. Biochem. J. 354, 275-283 (2001).

28. Oakhill, J. S. et al. beta-Subunit myristoylation is the gatekeeper for initiating metabolic stress sensing by AMP-activated protein kinase (AMPK). Proc. Natl Acad. Sci. USA 107, 19237-19241 (2010).

29. Davies, S. P., Sim, A. T. \& Hardie, D. G. Location and function of three sites phosphorylated on rat acetyl-CoA carboxylase by the AMP-activated protein kinase. Eur. J. Biochem 187, 183-190 (1990).

30. Winder, W. W. et al. Phosphorylation of rat muscle acetyl-CoA carboxylase by AMP-activated protein kinase and protein kinase A. J. Appl. Physiol. 82, 219-225 (1997).

31. Abu-Elheiga, L. et al. The subcellular localization of acetyl-CoA carboxylase 2. Proc. Natl. Acad. Sci. USA 97, 1444-1449 (2000). 
32. Abu-Elheiga, L., Oh, W., Kordari, P. \& Wakil, S. J. Acetyl-CoA carboxylase 2 mutant mice are protected against obesity and diabetes induced by high-fat/high-carbohydrate diets. Proc. Natl. Acad. Sci. USA 100, 10207-10212 (2003).

33. Wakil, S. J. \& Abu-Elheiga, L. A. Fatty acid metabolism: target for metabolic syndrome. J. Lipid Res. 50(Suppl), S138-S143 (2009).

34. Hardie, D. G., Ross, F. A. \& Hawley, S. A. AMPK: a nutrient and energy sensor that maintains energy homeostasis. Nat. Rev. Mol. Cell Biol. 13, 251-262 (2012).

35. Li, Y. et al. AMPK phosphorylates and inhibits SREBP activity to attenuate hepatic steatosis and atherosclerosis in diet-induced insulin-resistant mice. Cell Metab. 13, 376-388 (2011).

36. Qian, X. et al. Conversion of PRPS hexamer to monomer by AMPK-mediated phosphorylation inhibits nucleotide synthesis in response to energy stress. Cancer Discov. 8, 94-107 (2018).

37. Inoki, K., Zhu, T. \& Guan, K. L. TSC2 mediates cellular energy response to control cell growth and survival. Cell 115, 577-590 (2003).

38. Gwinn, D. M. et al. AMPK phosphorylation of raptor mediates a metabolic checkpoint. Mol. Cell 30, 214-226 (2008).

39. Zoncu, R. et al. mTORC1 senses lysosomal amino acids through an inside-out mechanism that requires the vacuolar $\mathrm{H}(+)$-ATPase. Science 334, 678-683 (2011).

40. Efeyan, A. et al. Regulation of mTORC1 by the Rag GTPases is necessary for neonatal autophagy and survival. Nature 493, 679-683 (2013).

41. Pehmoller, C. et al. Genetic disruption of AMPK signaling abolishes both contraction- and insulin-stimulated TBC1D1 phosphorylation and 14-3-3 binding in mouse skeletal muscle. Am. J. Physiol. Endocrinol. Metab. 297, E665-E675 (2009).

42. Vichaiwong, K. et al. Contraction regulates site-specific phosphorylation of TBC1D1 in skeletal muscle. Biochem. J. 431, 311-320 (2010).

43. Egan, D. F. et al. Phosphorylation of ULK1 (hATG1) by AMP-activated protein kinase connects energy sensing to mitophagy. Science 331, 456-461 (2011).

44. Kim, J., Kundu, M., Viollet, B. \& Guan, K. L. AMPK and mTOR regulate autophagy through direct phosphorylation of Ulk1. Nat. Cell Biol. 13, 132-141 (2011).

45. Kim, J. et al. Differential regulation of distinct Vps34 complexes by AMPK in nutrient stress and autophagy. Cell 152, 290-303 (2013).

46. Youle, R. J. \& van der Bliek, A. M. Mitochondrial fission, fusion, and stress. Science 337, 1062-1065 (2012)

47. Toyama, E. Q. et al. Metabolism. AMP-activated protein kinase mediates mitochondrial fission in response to energy stress. Science 351, 275-281 (2016).

48. Kim, N. et al. AMPKalpha2 translocates into the nucleus and interacts with hnRNP $\mathrm{H}$ : implications in metformin-mediated glucose uptake. Cell. Signal. 26, 1800-1806 (2014).

49. Wu, D. et al. Glucose-regulated phosphorylation of TET2 by AMPK reveals a pathway linking diabetes to cancer. Nature 559, 637-641 (2018).

50. Chia, I. V. \& Costantini, F. Mouse axin and axin2/conductin proteins are functionally equivalent in vivo. Mol. Cell. Biol. 25, 4371-4376 (2005).

51. Nusse, R. \& Clevers, H. Wnt/beta-catenin signaling, disease, and emerging therapeutic modalities. Cell 169, 985-999 (2017).

52. Corton, J. M., Gillespie, J. G., Hawley, S. A. \& Hardie, D. G. 5-aminoimidazole-4carboxamide ribonucleoside. A specific method for activating AMP-activated protein kinase in intact cells? Eur. J. Biochem 229, 558-565 (1995).

53. Faupel, R. P., Seitz, H. J., Tarnowski, W., Thiemann, V. \& Weiss, C. The problem of tissue sampling from experimental animals with respect to freezing technique, anoxia, stress and narcosis. A new method for sampling rat liver tissue and the physiological values of glycolytic intermediates and related compounds. Arch. Biochem. Biophys. 148, 509-522 (1972).

54. Dale, S., Wilson, W. A., Edelman, A. M. \& Hardie, D. G. Similar substrate recognition motifs for mammalian AMP-activated protein kinase, higher plant HMG-CoA reductase kinase-A, yeast SNF1, and mammalian calmodulin-dependent protein kinase I. FEBS Lett. 361, 191-195 (1995).
55. Scott, J. W. et al. Thienopyridone drugs are selective activators of AMP-activated protein kinase beta1-containing complexes. Chem. Biol. 15, 1220-1230 (2008).

56. Luo, W. et al. Axin contains three separable domains that confer intramolecular, homodimeric, and heterodimeric interactions involved in distinct functions. J. Biol. Chem. 280, 5054-5060 (2005).

57. Kim, S. E. et al. Wnt stabilization of beta-catenin reveals principles for morphogen receptor-scaffold assemblies. Science 340, 867-870 (2013).

58. Xin, F. J., Wang, J., Zhao, R. Q., Wang, Z. X. \& Wu, J. W. Coordinated regulation of AMPK activity by multiple elements in the alpha-subunit. Cell Res. 23, 1237-1240 (2013).

59. $\mathrm{Li}, \mathrm{X}$. et al. Structural basis of AMPK regulation by adenine nucleotides and glycogen. Cell Res. 25, 50-66 (2015).

60. Ross, F. A., MacKintosh, C. \& Hardie, D. G. AMP-activated protein kinase: a cellular energy sensor that comes in 12 flavours. FEBS J. 283, 2987-3001 (2016).

61. Olivier, S., Foretz, M. \& Viollet, B. Promise and challenges for direct small molecule AMPK activators. Biochem. Pharmacol. 153, 147-158 (2018).

62. Khan, A. S. \& Frigo, D. E. A spatiotemporal hypothesis for the regulation, role, and targeting of AMPK in prostate cancer. Nat. Rev. Urol. 14, 164-180 (2017).

63. Boudaba, N. et al. AMPK re-activation suppresses hepatic steatosis but its downregulation does not promote fatty liver development. EBioMedicine $\mathbf{2 8}$ 194-209 (2018).

64. Cao, Y. et al. Activation of gamma2-AMPK suppresses ribosome biogenesis and protects against myocardial ischemia/reperfusion injury. Circ. Res. 121, 1182-1191 (2017).

65. Woods, A. et al. Liver-specific activation of AMPK prevents steatosis on a highfructose diet. Cell Rep. 18, 3043-3051 (2017).

66. Su, H. et al. VPS34 acetylation controls its lipid kinase activity and the initiation of canonical and non-canonical autophagy. Mol. Cell 67, 907-921 e907 (2017).

67. Cheong, H., Lindsten, T., Wu, J., Lu, C. \& Thompson, C. B. Ammonia-induced autophagy is independent of ULK1/ULK2 kinases. Proc. Natl Acad. Sci. USA 108, 11121-11126 (2011).

68. Soeters, M. R., Soeters, P. B., Schooneman, M. G., Houten, S. M. \& Romijn, J. A Adaptive reciprocity of lipid and glucose metabolism in human short-term starvation. Am. J. Physiol. Endocrinol. Metab. 303, E1397-E1407 (2012).

69. Cahill, G. F. Jr. Starvation in man. Clin. Endocrinol. Metab. 5, 397-415 (1976).

70. Pilegaard, H., Saltin, B. \& Neufer, P. D. Effect of short-term fasting and refeeding on transcriptional regulation of metabolic genes in human skeletal muscle. Diabetes 52, 657-662 (2003).

71. Li, T. Y. et al. ULK1/2 constitute a bifurcate node controlling glucose metabolic fluxes in addition to autophagy. Mol. Cell 62, 359-370 (2016).

72. Abe, Y. et al. Mouse model of liver ischemia and reperfusion injury: method for studying reactive oxygen and nitrogen metabolites in vivo. Free Radic. Biol. \& Med. 46, 1-7 (2009).

73. Wieckowski, M. R., Giorgi, C., Lebiedzinska, M., Duszynski, J. \& Pinton, P. Isolation of mitochondria-associated membranes and mitochondria from animal tissues and cells. Nat. Protoc. 4, 1582-1590 (2009).

74. Kobayashi, T. et al. Separation and characterization of late endosomal membrane domains. J. Biol. Chem. 277, 32157-32164 (2002).

75. Zhao, J. et al. Study of polar metabolites in tobacco from different geographical origins by using capillary electrophoresis-mass spectrometry. Metabolomics 10, 805-815 (2014).

76. Zhao, Y. et al. A metabolomics study delineating geographical locationassociated primary metabolic changes in the leaves of growing tobacco plants by GC-MS and CE-MS. Sci. Rep. 5, 16346 (2015).

77. Wang, S., Wang, Z., Zhou, L., Shi, X. \& Xu, G. Comprehensive analysis of short-, medium-, and long-chain acyl-coenzyme a by online two-dimensional liquid chromatography/mass spectrometry. Anal. Chem. 89, 12902-12908 (2017). 